\title{
Von der „Zigeunerwarntafel“ zum Verbrecherbild. Eine historisierende Perspektive auf die Kriminalisierung von Sinti_ze und Rom_nja im deutschsprachigen Raum
}

\author{
Laura Soréna Tittel
}

\section{Abstract}

This contribution analyses continuity and change in the representation of Sinti and Roma used by the police in German-speaking countries over several centuries. Drawing on iconographic studies of visual antiziganism, it examines images produced by the police as an expression of a state securitisation practice. Whereas in the 17th and 18th centuries the threat of punishment and the exercise of state power was enacted as a spectacle directly within the image itself, this changed in the 19th century with the emergence of photography as a police technology. For the period between 1850 and 1937, we can trace a development from photographic experimentation to internationally standardised photographs of (suspected) criminals. This contribution concludes that the once open threat of punishment continued to persist in a more subtle form of criminalisation and in the threat of social exclusion that this involved. It further shows that the state institutions used the images to present themselves as powerful and strong, initially directly by displays of state power within the image itself and later by exercising their power to set the framework.

\section{Einleitung}

Bis heute nutzen staatliche Institutionen in Deutschland das antiziganistisch geprägte Bild des kriminellen „Zigeuners“, um Maßnahmen gegenüber Sinti_ze und Rom_nja, aber auch Migrant_innen aus ost- und südosteuropäischen Ländern durchzusetzen. ${ }^{1}$ Die Verdrängung aus den

1 Der Begriff „Zigeuner“ wird im Beitrag als Quellenbegriff benutzt, wenn er sich ausschließlich auf die fiktive Vorstellung derjenigen, die ihn in abwertender Absicht benutzt haben, bezieht. Wenn es um reale Personen geht, wie sie beispielsweise in Fotografien vorkommen, werden die Begriffe Sinti_ze, Rom_nja oder Jenische benutzt, obwohl aus heutiger Perspektive in vielen Fällen nicht sicher nachvollzogen werden kann, ob die jeweiligen Personen tatsächlich Angehörige einer dieser Minderheiten waren oder aus anderen Gründen als „Zigeuner“ oder „Zigeunerinnen“ behandelt wurden. 
Innenstädten steht ebenso auf der Tagesordnung wie Abschiebungen, rassistische Polizeikontrollen und behördliche Schikane. ${ }^{2}$ Damit knüpft die heutige Politik an eine lange Tradition antiziganistischer Praktiken der Ausgrenzung, Verdrängung und Stigmatisierung von staatlicher Seite an, von denen im deutschsprachigen Raum zumeist Sinti_ze, Rom_nja und Jenische betroffen waren und sind.

Dieser Beitrag untersucht Bilder aus vier Jahrhunderten, auf denen „Zigeuner" und „Zigeunerinnen“ dargestellt sind und die zu polizeilichen Zwecken eingesetzt wurden. Aus medienanalytischer Sicht lässt sich zwischen Gemälden, druckgraphischen Erzeugnissen und Fotografien unterscheiden. In den historisch früheren gemalten Bildern sind überwiegend fiktive Figuren zu sehen, die „Zigeuner" oder „Zigeunerinnen“ darstellen sollen. Die modernen Fotografien hingegen zeigen tatsächliche Individuen, die mutmaßlich den Minderheiten der Sinti_ze, Rom_nja und Jenischen zugehören. Der ohnehin vorhandene Grundunterschied zwischen gemalten Bildern, die eher das Ergebnis von Herstellungs- als von Abbildungsprozessen sind, und Fotografien, die leibhaftige Modelle ablichten und dadurch in einem engeren mimetischen Verhältnis zu ihnen stehen, korreliert mit der Differenz zwischen dem „Zigeunerbild“ als Imagination und den konkreten Bildern von als „Zigeuner“ stigmatisierten Sinti_ze, Rom_nja und Jenischen. Während die Zurichtung des "Zigeunerbilds“ im Gemälde offensichtlich ist, bedarf es bei Fotografien einer größeren Anstrengung, um das gedankliche Bild hinter der Abbildung aufzudecken und einzuordnen. Der Beitrag geht auf diese unterschiedlichen Formen der Darstellung ein und verfolgt die These, dass Bilder von staatlicher Seite über Jahrhunderte dazu genutzt wurden, Menschengruppen voneinander abzugrenzen, sie als unterschiedlich bedrohlich darzustellen und vermeintliche Differenzen festzuschreiben. Die zu polizeilichen Zwecken hergestellten Bilder werden als Ausdruck einer staatlichen Praxis untersucht, die durch visuelle Versicherheitlichung Macht und Herrschaft aufbaut und erhält.

Die Antiziganismusforschung verhandelt Antiziganismus häufig als ein Problem der Einstellung bzw. als ein Konglomerat von Vorurteilen, die in der Bevölkerung kursieren. ${ }^{3}$ Auf die Vorurteilsforschung aufbauend untersucht sie Bilder und tradierte Stereotype des „Zigeuners“, wobei so-

2 Vgl. etwa die Dokumentation von Vorfällen in Berlin für das Jahr 2017 in: Amaro Foro e.V. 2018.

3 Vgl. die zahlreiche Literatur, die bereits im Titel von Vorurteilen spricht, u.a. Giere 1996; Mappes-Niediek 2013; Agarin 2014; Benz 2014; Bell 2015; Wippermann 2015. 
wohl metaphorische Bilder in der Literatur als auch tatsächliche bildliche Darstellungen herangezogen werden. ${ }^{4}$ Dieser vorurteilsbezogene Ansatz ist wichtig, um die Inhalte antiziganistischen Denkens zu verstehen. Um zu erklären, wie die Vorurteile und Stereotype mit diskriminierenden Praktiken zusammenhängen, ist eine weitergehende Untersuchung von gesellschaftlichen, politischen und rechtlichen Strukturen und daraus resultierenden Handlungen und routiniertem Verhalten - etwa bei Behörden - unabdingbar. ${ }^{5}$ Hier setzt der vorliegende Beitrag an, indem die betreffenden Bilder auf die Entwicklung von Macht- und Herrschaftsstrukturen hin untersucht werden.

Mit den politischen und rechtlichen Rahmenbedingungen antiziganistischer Verfolgung haben sich bereits einige historische Studien beschäftigt. Insbesondere für den polizeilichen Kontext sind hier die Studien von Leo Lucassen (1996), Wim Willems (1997) und Jennifer Illuzzi (2014) zu nennen, die sich mit dem "Zigeuner"-Begriff bei der Polizei befassen. Die Bildforschung behandelt besonders die Ikonografie antiziganistischer Stereotype und die Herstellung oder Inszenierung des „Anderen“. Der Historiker Frank Reuter hat sich eingehend mit der fotografischen Darstellung von Sinti_ze und Rom_nja beschäftigt und liefert einen umfassenden Überblick zum Thema. Seine Arbeiten fokussieren die Herstellung, Inszenierung und Anziehungskraft des „Fremden“ und suchen nach den Einflüssen der Fotografie auf die Genese der "Zigeuner"-Stereotype (vgl. Reuter 2011, 2014, 2016, 2019).6 Damit bewegen sie sich überwiegend im Bereich der Einstellungs- und Vorurteilsforschung, während die historischen Studien zum „Zigeuner"-Begriff immer wieder das Verhältnis von sozialer Frage und Rassismus in der Polizeigesetzgebung untersuchen (vgl. auch Meuser 2014; Tittel 2020).

Der vorliegende Beitrag baut auf diesen wichtigen Untersuchungen auf und ergänzt sie um die visuelle Dimension der strukturellen und institutionellen Diskriminierung und der Verfolgungspraktiken von staatlicher Seite. Dazu werden allgemeine Studien zur Entwicklung von Polizeibil-

4 Zu metaphorischen Bildern vgl. etwa Solms 2008; zur Analyse von Stereotypen in bildlichen Darstellungen vgl. Bell 2015.

5 Vgl. zur Kritik an reiner Vorurteilsforschung Koch (2010, S. 255). Ein gelungenes Beispiel zu aktuellem Antiziganismus in den Behörden ist die Untersuchung von End (2019), die sich dem Thema u.a. durch die Analyse von Pressemitteilungen nähert.

6 Vgl. auch Holzer (2008) für einen Überblick über die Mechanismen der exotisierenden Inszenierung des „Zigeuners“, insbesondere durch Fotografien aus Ostund Südosteuropa. 
dern und erkennungsdienstlicher Fotografie herangezogen, wie sie bei Susanne Regener (1999) und Jens Jäger (2009) zu finden sind. Zudem wird eine gesellschaftskritische Perspektive eingenommen, die sich auf Susan Sontags Studien zur Fotografie im Kontext einer kapitalistischen Gesellschaftsordnung (1987) und Michel Foucaults (1989) Überlegungen zum Wandel von Strafpraktiken in der Moderne bezieht, um visuellen Antiziganismus als Herrschaftsform und -praxis zu analysieren. Der Fokus liegt auf dem Einsatz von Bildern durch die Polizei im Rahmen der Versicherheitlichung von Sinti_ze, Rom_nja und Jenischen. Die Zeitspanne reicht vom 17. bis ins 20. Jahrhundert. Vier Fallbeispiele von der gemalten "Zigeunerwarntafel" über Darstellungen von Gerichtsprozessen zu den modernen Techniken der Polizeifotografie dienen als Schlaglichter, anhand derer die historische Transformation visueller Praktiken von Versicherheitlichung aufgezeigt wird.

\section{Strafandrohung und staatliche (Selbst-)Inszenierung in frühneuzeitlichen Gemälden und Druckgrafiken mit „Zigeuner"-Figuren}

Die Anfang des 18. Jahrhunderts in Europa weit verbreiteten „Zigeunerwarntafeln“ waren ab etwa 1685 vielerorts auch unter den Namen „Heiden-“, „Zigeuner-“ oder „Vagabundenstöcke“, „Tatern-“ oder „Heidenpfähle" aufgekommen. ${ }^{7}$ Sie zielten trotz der unterschiedlichen Bezeichnungen allesamt auf die Abschreckung von "Zigeunern“ und „Zigeunerinnen“. Für meine Analyse antiziganistisch eingesetzter Bilder dienen sie als Vorläufer der späteren Polizeifotografie, da beide Formate "Zigeuner" in eine Sonderstellung brachten: Warntafeln dieser Art für andere Bevölkerungsgruppen sind nicht bekannt, ebenso wie zu Beginn des 20. Jahrhunderts keine andere soziale Gruppe von der Polizei so umfassend fotografisch festgehalten wurde. ${ }^{8}$ In den frühneuzeitlichen gemalten und gedruckten

7 Für weitere Details zu den Jahreszahlen und der Verbreitung vgl. Steiner (2019, S. 142). In Einzelfällen wurde auch schon von früheren Tafeln berichtet, etwa durch den Heimat- und Geschichtsverein Elze im Kreis Springe, der Quellen gefunden hat, die zeigen, dass bereits 1635 durch den dort ansässigen Herzog sogenannte „Taternpfähle“ als Hinweisschilder an Grenzübergängen aufgestellt wurden, vgl. Wieckhorst 2012.

8 Vom Medienformat der Porträtfotografie her gedacht wäre hier zunächst an gemalte Porträts oder wortgestützte Personenbeschreibungen zu denken. Letztere gab es in der Frühen Neuzeit auch von als „Zigeuner“ eingeordneten Menschen, vgl. etwa Jeutter 2016, S. 34; allerdings nahmen "Zigeuner“ in diesem Format keine Sonderstellung ein. 
Inszenierungen von „Zigeunern“ und "Zigeunerinnen“ sind sowohl die Strafen und Bestrafungsinstrumente als auch verschiedene Motive erkennbar, welche auf die kirchliche und weltliche Ordnung verweisen. Durch die Strafandrohung wird markiert, dass die zu Bestrafenden sich unrechtmäßig (kriminell) verhalten und somit eine Bedrohung darstellen, die ferngehalten werden soll. Anders als in den späteren Polizeifotografien ist die Obrigkeit im Bild direkt repräsentiert und werden die drohenden Strafen explizit dargestellt.

Bei den Warntafeln handelt sich um gemalte oder im Holzschnittverfahren gedruckte Bilder, die meist mit einem kleinen Text versehen und auf Holz- oder Metalltafeln im öffentlichen Raum angebracht waren. Auf den Tafeln wurden die angedrohten Strafen für einen verbotenen Grenzübertritt, die auf Verordnungen zur Ausweisung von „Zigeunern“ basierten, eindrücklich vor Augen geführt. Die bildliche Darstellung hatte den Vorteil, dass Analphabetismus nicht mehr als Ausrede für die Unkenntnis der Regelungen gelten konnte (vgl. Steiner 2019, S. 141). Um direkt vor den Konsequenzen des Grenzübertritts zu warnen, wurden die Tafeln, ähnlich Straßenschildern, an Grenzübergängen, Pässen und Ortseingängen aufgestellt. Einige wenige Exemplare haben sich bis heute erhalten und finden sich in Museen in Deutschland, Österreich, den Niederlanden und Tschechien (vgl. Steiner 2019, S. 140). Die Ausbreitung erstreckte sich mindestens über Mitteleuropa. Eine genaue Anzahl lässt sich nicht rekonstruieren, jedoch kann von einer sehr dichten Verteilung ausgegangen werden, da beispielsweise für das Jahr 1712 die Herstellung von 124 Warntafeln allein in Württemberg nachgewiesen ist (vgl. Fricke 1996, S. 206-207). Über den konkreten Fertigungsprozess der Warntafeln ist wenig bekannt. Einzelne Bildvorlagen für den Druck, wie ein Exemplar aus Schlesien von 1708, sind noch erhalten (vgl. Härter 2005, S. 69).

Eine der überlieferten gemalten Warntafeln stammt aus dem späten 17. oder frühen 18. Jahrhundert und wird im Volkskundemuseum in Graz aufbewahrt (Abb. 1). ${ }^{9}$ Anders als andere erhaltene Exemplare, die nur eine einzelne Szene darstellen, zeigt sie mehrere Bestrafungsszenen im Bildraum verteilt und detailliert ausgeführt. Der Schriftzug, der das Bild am oberen und unteren Rand rahmt, besagt in Form eines Reimes: „Lost Ihr Zügäiner, Alchier bleib kheiner / Auß dem Landt Thuet Weichen, Sonst wird Man Euch Außstreichen“ (= „Los ihr Zigeuner, hier bleibt kei-

9 Hierzu gibt es widersprüchliche Angaben: Im Sammelband von Uerlings et al. (2011, S. 380-381) heißt es, die Tafel stamme aus dem 17. Jahrhundert, während Steiner (2019, S. 141) die Tafel auf die erste Hälfte des 18. Jahrhunderts datiert. 


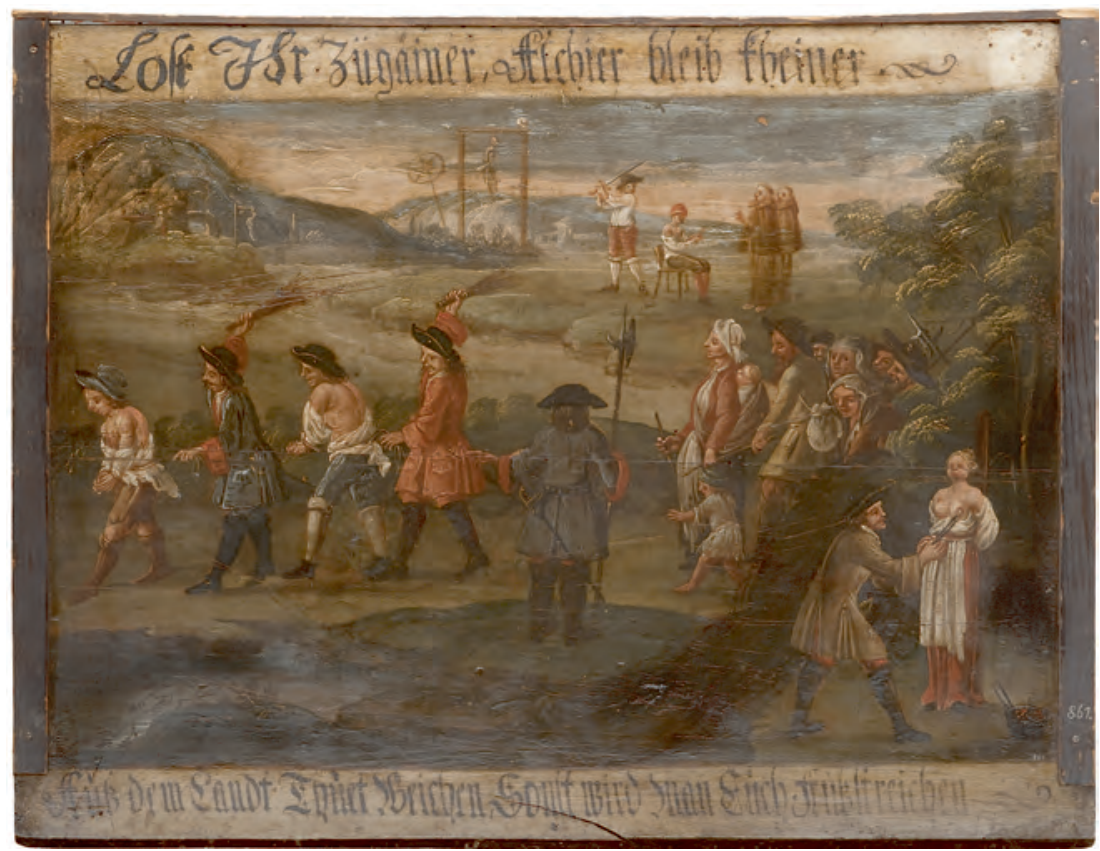

Abb. 1: „Zigeunerverbotstafel“, 17. oder 18. Jahrhundert, Öl auf Holz, 52,2 x $67,5 \mathrm{~cm}$ (Universalmuseum Joanneum/Sammlung Volkskundemuseum Graz, Inv. 35.867)

ner, aus dem Land tut weichen, sonst wird man euch ausstreichen [=auspeitschen] "). ${ }^{10}$ Der Text spricht „Zigeuner" direkt an und ergänzt mit den schriftlichen Aufforderungen und Androhungen die bildliche Darstellung des Strafvollzugs. Gezeigt werden zu jener Zeit übliche Strafen wie Verletzungen durch Brandmarkungen, Auspeitschen, Rädern, Enthauptung und das Erhängen am Galgen.

Für die bildliche Darstellung wurden Szenen mit mehreren Figuren gemalt, deren Status oder Zugehörigkeit durch äußerliche Merkmale wie Haltung, Blick, Kleidung und Accessoires markiert wird. Eine Unterscheidung durch Haut- oder Haarfarbe, wie sie ab dem Zeitalter der Aufklärung wichtig wurde, ist in den frühen Bildern nicht zu finden. ${ }^{11}$ Die in der

10 Eigene Übersetzung.

$11 \mathrm{Ab}$ den 1780er Jahren, besonders mit Erscheinen des Buches Die Zigeuner von Heinrich Moritz Gottlieb Grellmann (1783), verbreitete sich die These, dass die „Zigeuner“ aus Indien kämen. Damit wurde auch das Bild des „Zigeuners“ zu- 
Grazer Warntafel dargestellten Personen lassen sich in drei Gruppen einteilen: Die Strafenden, die potentiell zu Bestrafenden und die direkt im Bild Bestraften. Zu den Strafenden gehören Repräsentanten der weltlichen Obrigkeit und der Kirche. Die Vertreter der Obrigkeit tragen edle Hüte und Mäntel und haben Gegenstände zur Bestrafung in der Hand, die von Peitschen über eine glühende Zange und ein Schwert bis zu einer Hellebarde, einer frühneuzeitlichen Hieb- und Stichwaffe, reichen. Zwei Mönche wohnen - als Repräsentanten der Kirche und somit auch als moralische Instanz - einer Enthauptungsszene im Hintergrund des Bildes bei.

Als potentiell zu Bestrafende kann eine Gruppe von Menschen ausgemacht werden, die rechts im Bild versammelt sind. Sie stehen vor einem Grenzübergang, der durch einen zentral im Bild platzierten Staatsdiener mit Hellebarde in Rückenansicht gekennzeichnet ist. Dieser zeigt auf eine Szene zu seiner linken Seite, in der zwei Männer durch Vertreter der Strafjustiz ausgepeitscht werden. Somit wird der ankommenden Gruppe ihr Schicksal gezeigt, sollte sie den Grenzübertritt wagen. Den potentiell zu Bestrafenden wird also auf bildlicher Ebene die Wahl gelassen, nun noch umzukehren. Sie stehen dicht an dicht gedrängt und blicken in Richtung der Auspeitschungsszene. Ihre Darstellung als gedrängte Gruppe inklusive einer Mutterfigur mit mehreren Kindern gehört zu der sich herausbildenden antiziganistischen Ikonografie. ${ }^{12}$ In dieser findet sich bereits eine Verbindung der Darstellung von Räuberbanden mit angeblichem Kinderreichtum, die sich bis heute im Stereotyp der kriminellen Clans als Großfamilien gehalten hat. ${ }^{13}$ Die Gruppenmitglieder werden mit einfacher Stoffkleidung dargestellt und verschwinden auf der rechten Seite beinahe im Schatten des dunklen Gebüschs.

Die direkt Bestraften sind hingegen wenig bekleidet. Sie haben durchweg entblößte Oberkörper, sei es zum Auspeitschen, zur Brandmarkung oder für die Enthauptung. Ihre nackten Oberkörper und ihre gebeugte Haltung stehen im Kontrast zu den uniformierten und aufrechten Repräsentanten der Ordnungsmacht. ${ }^{14}$ Im Vordergrund rechts wird sehr promi-

nehmend rassialisiert, Haar- und Hautfarbe wurden zu wichtigen Abgrenzungsmerkmalen (vgl. Tittel 2020, S. 7-8).

12 Mehr hierzu bei Reuter (2014, S. 89), der bezeugt, dass sich das Motiv des „Zigeunerzugs" seit Ende des 15. Jahrhunderts in der Kunst nachweisen lässt.

13 Mehr zur Ikonografie und dem Motiv des Kinderreichtums bei Bell 2015, S. 158.

14 Siehe zur Ikonografie einer ähnlichen Auspeitschungsszene auf einer anderen „Zigeunerwarntafel“ die Interpretation von Reuter 2014, S. 70: „Augenfällig ist das Gegensatzpaar des entblößten ,Zigeuners', an dem die Strafe vollzogen wird, und 
nent eine an einen Pfahl gebundene Frau mit entblößtem Oberkörper von einem Vertreter der Obrigkeit mit glühenden Zangen an den Brüsten gebrandmarkt, wie ein Topf mit glühenden Kohlen verdeutlicht. Im Hintergrund sind Galgen mit Gehenkten in wehenden Stofffetzen sowie ein Holzrad zum Rädern zu erkennen.

Von der Androhung der Strafen auf den Warntafeln kann nicht direkt auf die Durchführung der Bestrafung bei Grenzübertritt geschlossen werden. Die Umsetzung und Wirkung der frühneuzeitlichen Gesetzgebung werden in der Forschung kontrovers diskutiert (vgl. Härter 2005, S. 66). Im Bereich der Versicherheitlichung von Sinti_ze und Rom_nja ist ebenso wie in anderen Bereichen davon auszugehen, dass die Normen und Verordnungen nicht vollständig, aber doch teilweise umgesetzt wurden (vgl. Härter 2005, S. 66; Landwehr 2001, S. 59).

Ein Beispiel für tatsächliche Bestrafung, welches sogleich selbst wiederum durch visuelle Darstellung zur Abschreckung nutzbar gemacht wurde, ist ein Gerichtsprozess von 1726. Bei diesem Prozess wurde eine angebliche "Zigeunerbande“ vor das „Peinliche Gericht" im mittelhessischen Gießen gestellt, welches für Strafen an Leib und Leben (sogenannte „peinliche Strafen“) zuständig war. 25 der 28 Angeklagten wurden zum Tode verurteilt, darunter acht Frauen. Bereits für die bloße Anwesenheit drohte ihnen als "Zigeuner" und "Jauner" laut einer hessischen Verordnung von 1722 bei erstmaligem Aufgreifen eine Brandmarkung und bei erneutem Aufgreifen die Todesstrafe, „wann auch sonsten weiter keine speziale Missethat auff sie gebracht werden könnte“ (Auszug aus der Poenal-Sanction von 1722 in: Hessisches Kultusministerium 2015, S. 33). Den Angeklagten wurde jedoch nicht nur ihre Anwesenheit auf dem Territorium vorgeworfen, sondern auch zahlreiche Delikte wie Diebstahl, Raubüberfall und Mord. Ob die Anschuldigungen gerechtfertigt waren, soll an dieser Stelle nicht weiter erörtert werden. ${ }^{15}$ Die vorliegenden Materialen machen deutlich, dass das Verfahren als ein Prozess an „Zigeunern" stilisiert wurde und der Abschreckung dienen sollte.

des uniformierten Vertreters der Ordnungsmacht, repräsentiert durch den Schafsrichter." Auf S. 71 geht Reuter kurz in ähnlicher Weise auch auf die Grazer Tafel ein.

15 Manfred-Guido Schmitz (2011, S. 140-153) kommt in seiner eher populärwissenschaftlichen Untersuchung des Prozesses zum Schluss, dass es im Verfahren nicht um die Aufklärung der Straftaten ging, sondern um politische Interessen, und dass letztendlich alle Angeklagten unschuldig hingerichtet wurden bzw. außer einem Hühnerdiebstahl keine einzige Tat nachgewiesen werden konnte. 
Dies zeigt insbesondere das Buch Ausfübrliche Relation Von der Famosen Ziegeuner-,Diebs-, Mord- und Rauber-Bande, Welche Den 14. und 15. Novembr. Ao. 1726. zu Giessen durch Schwerdt, Strang und Rad, respective justificirt worden [...], das 1727 von Dr. Johann Benjamin Weissenbruch veröffentlicht wurde. ${ }^{16}$ Der Verfasser war als Gerichts-Assessor am „Peinlichen Gericht zu Giessen“ selbst am Prozess beteiligt gewesen und vom Landgrafen Ernst Ludwig von Hessen mit dem Buch beauftragt worden. Weissenbruch beschreibt den Prozesshergang und versucht, die Strafen zu rechtfertigen. Er beginnt mit einer ausführlichen Erklärung dessen, wer und was „Zigeuner" seien, geht dabei auf verschiedene sprachliche Herleitungen des Begriffs ein und zitiert diverse Theorien aus dem 17. und 18. Jahrhundert über die Herkunft und Geschichte der "Zigeuner" (vgl. Weissenbruch 1727, S. 3-8). Zudem erörtert er in einem eigenen Kapitel, „[o]b die Ziegeuner in einer Republic zu dulten" seien (ebd., S. 19-42). Dies verdeutlicht nochmals die politische Dimension des Strafprozesses. Die prominente Stellung dieser Vorüberlegungen als Einleitung in das Prozessgeschehen zeigt an, dass der Leserschaft von Anfang an verdeutlicht werden sollte, dass die Straftaten von einer bestimmten Gruppe begangen wurden. So wurde der Eindruck vermittelt, es gäbe eine Verbindung zwischen der angeblichen Herkunft der Angeklagten und den vorgeworfenen Straftaten.

Weissenbruchs Buch ist durch mehrere Bilder illustriert, die den Verbrechenshergang und die Bestrafung darstellen. Eines zeigt die Szene der Urteilsvollstreckung, welche als Spektakel vor großem Publikum inszeniert wird (Abb. 2). Deutlich zu sehen sind die unterschiedlichen Arten der Hinrichtung, die den 25 Verurteilten widerfuhren: Links im Bild sind auf einem vom Publikum umringten Platz Enthauptungen durch das Schwert zu sehen. Außerdem wird eine Räderung gezeigt, durch welche laut der Beschreibung im Buch die vermeintlichen Bandenanführer hingerichtet wurden. In einer Art Graben liegen Leichen mit abgetrennten Köpfen, Männer wie Frauen. Auf der rechten Seite des Bildes, abgegrenzt auf einem weiteren Schauplatz, sind sowohl Gestelle mit Rädern und aufgespießten Köpfen als auch eine große Anzahl an Erhängten an einem Gerüst zwischen drei Galgentürmen zu sehen. An allen Schauplätzen der Hinrichtungen sind Vertreter der Obrigkeit anwesend, die die Urteilsvollstreckungen begleiten und dokumentieren. Sie heben sich durch ihre

16 Das Buchformat „Relation“ kam ab 1700 in Mode, um über spektakuläre Straftaten und Gerichtsprozesse zu berichten. So veröffentlichte das Reichskammergericht in dieser Zeit zahlreiche eigens angefertigte „Relationen“. Vgl. Jeutter 2016, S. 36 . 


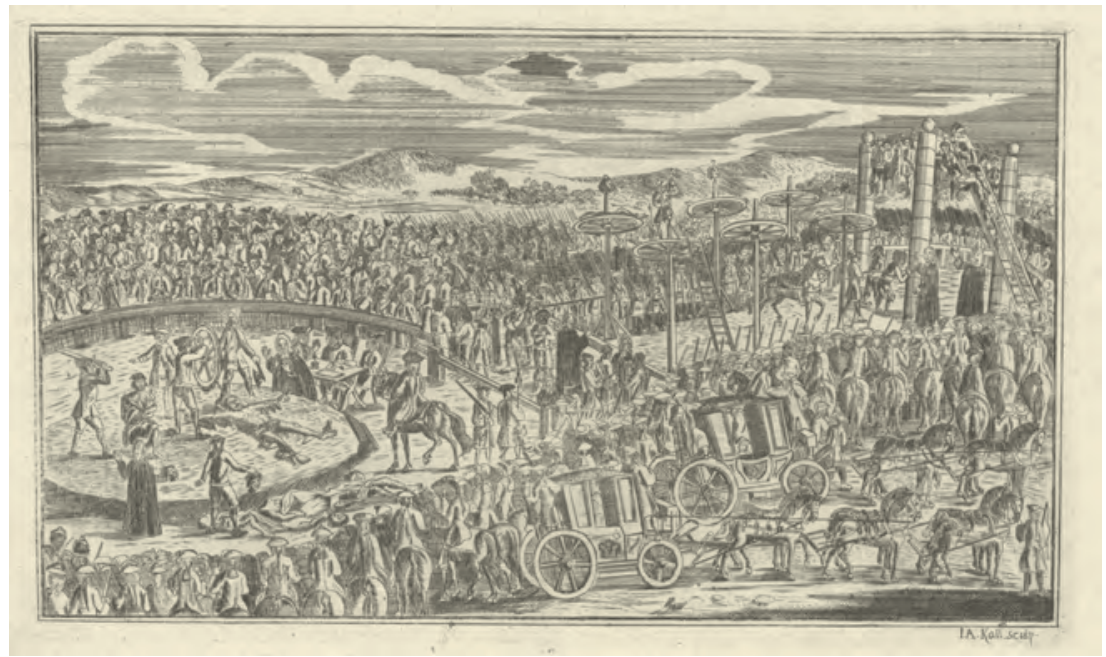

Abb. 2: Johann Andreas Kall, Hinrichtung einer „Zigeunerbande“, o.J., Kupferstich, aus: Weissenbruch 1727 (SLUB Dresden, Digitale Sammlungen)

Kleidung in Form von Hüten und mit zahlreichen Knöpfen bestückten Mänteln, aber auch durch ihre Tätigkeiten - Schreiben, Anweisen oder Patrouillieren - ab.

Die gezeigten Todesstrafen gehörten ebenso wie das Auspeitschen und die Brandmarkung zur Kategorie der körperlichen Strafen, die für die Frühe Neuzeit charakteristisch waren. Die Darstellung der Bestrafungen als Spektakel hatte, ähnlich wie die zeitgenössischen "Zigeunerwarntafeln“, eine warnende Funktion. Beide Medien visualisieren die drastischen Strafen und inszenieren die Repräsentanten des Staates im Bild. Als Buch veröffentlicht, sollte der Prozess auch über das konkrete Ereignis 1726 in Gießen hinauswirken, sowohl als Abschreckung als auch als Vorbild für weitere Prozesse.

Die Entwicklung der frühen Polizeifotografie in der Schweiz

Während im 17. und 18. Jahrhundert gemalte „Zigeunerwarntafeln“ an Grenzübergängen die Strafen für einen verbotenen Übertritt visualisierten und Buchillustrationen den öffentlichen Strafvollzug zeigten, kam im 19. Jahrhundert mit der Fotografie ein Medium auf, das von der Polizei in besonderem Maße an „Zigeunern und nach Zigeunerart Umherziehenden“ 
erprobt wurde. Dies begann ab etwa 1850 mit der fotografischen Erfassung der sogenannten „Heimatlosen“ in der Schweiz und setzte sich im 1899 gegründeten Münchener „Nachrichtendienst für die Sicherheitspolizei in Bezug auf Zigeuner" fort. ${ }^{17}$

Die vermutlich älteste noch erhaltene Serie von Polizeifotografien datiert von 1852/53 und wurde von Carl Durheim (1810-90) im Berner Gefängnis aufgenommen (vgl. Meier/Wolfensberger 1998a, S. 9). Die Fotografierten waren infolge einer Fahndungsaktion auf Grundlage des schweizerischen Heimatlosengesetzes von 1850 inhaftiert worden, welches zwei Jahre nach der Gründung des schweizerischen Bundesstaats verabschiedet worden war. Die wochenlangen Verhaftungen dienten zunächst dem Ziel der Identitätsfeststellung. Im Zuge dessen wurde auch ausgehandelt, wer überhaupt als Schweizer_in gelten und das Gemeinde- oder Kantonsbürgerrecht erhalten konnte (vgl. Meier 2007, S. 227). Bei den abgelichteten Personen handelte es sich um sogenannte heimat- und staatenlose Personen, die für die Behörden nicht zuordenbar waren. Unter ihnen befanden sich zahlreiche Personen, deren Nachfahren später als Jenische bezeichnet wurden (vgl. Meier/Wolfensberger 1998a, S. 11), die wiederum im 20. Jahrhundert von der sogenannten Schweizerischen „Zigeunerpolitik“ betroffen waren (vgl. Huonker/Ludi 2001, S. 38-45; Meier/Wolfensberger 1998b, S. 383-494). Die festgenommenen Personen sollten in einem zweiten Schritt eingebürgert werden, wozu sie zwangsangesiedelt und zur Arbeit gezwungen wurden. Das Heimatlosengesetz zielte somit nicht nur auf die Abschaffung der Heimatlosigkeit, sondern richtete sich direkt gegen eine mobile Lebensweise (vgl. Meier 2007, S. 228). Für den Fall, dass die Zwangsansiedlung scheiterte, sollten die Fotografien dazu dienen, die Abgelichteten bei einem erneuten Aufgreifen wiederzuerkennen.

Heute sind 220 Salzpapierabzüge aus der Sammlung der Fahndungsbilder sowie ein Album mit 228 Lithografien, welche auf Nachzeichnungen der Fotografien basieren, im Schweizerischen Bundesarchiv archiviert. Durheims erste Aufnahmen waren im Oktober 1852 noch im Daguerreotypie-Verfahren entstanden und sind nicht überliefert (vgl. Meier/Wolfensberger 1998a, S. 14). Die Daguerreotypie war ein in der Zeit gängiges fotografisches Verfahren, welches die Aufnahmen auf einzelnen Metallplatten festhielt und keine Vervielfältigung zuließ. Salzpapierabzüge boten hingegen als erstes Negativ-Positiv-Verfahren in der Geschichte der Fotografie die Möglichkeit, beliebig viele Abzüge ohne Qualitätsverlust am Negativ herzustellen. Die Herstellung von Abzügen war jedoch sehr kostspielig,

17 Zum Aufbau des Nachrichtendienstes vgl. Albrecht 2002, S. 58-68. 
weshalb im Falle der schweizerischen Fahndungsfotografie zur Weiterverbreitung der Bilder an die Polizeistellen der verschiedenen Kantone Lithografien verwendet wurden (vgl. ebd., S. 12).

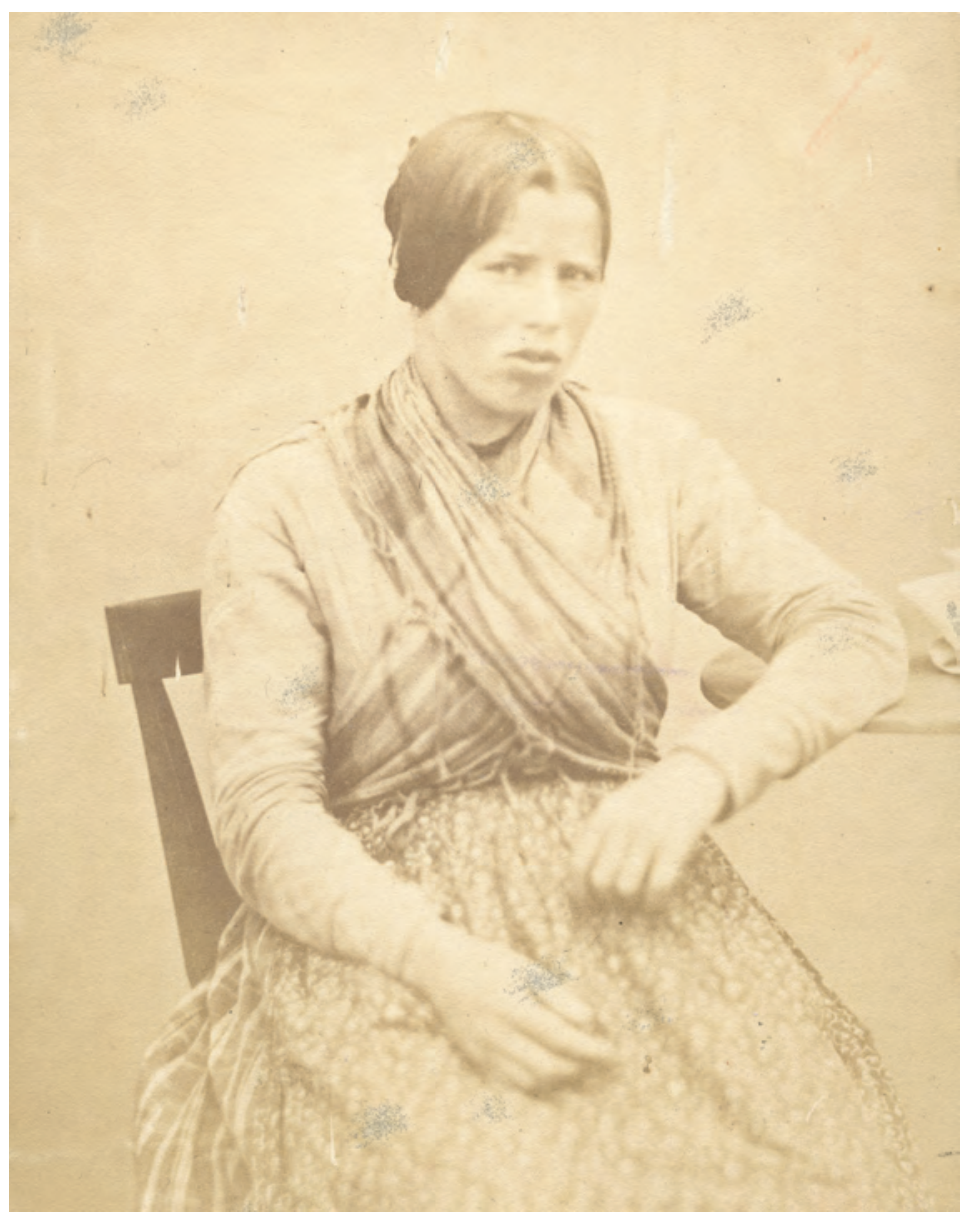

Abb. 3: Carl Durheim, Brutschi, Barbara, alias Barbara Pfister [geboren 1835, Beihälterin des Konrad Schneider], 1852/53, Fotografie, Salzpapierabzug, $10 \times 12,5 \mathrm{~cm}$ (Schweizerisches Bundesarchiv, Bern, CH-BAR 30313843; Wikimedia Commons) 


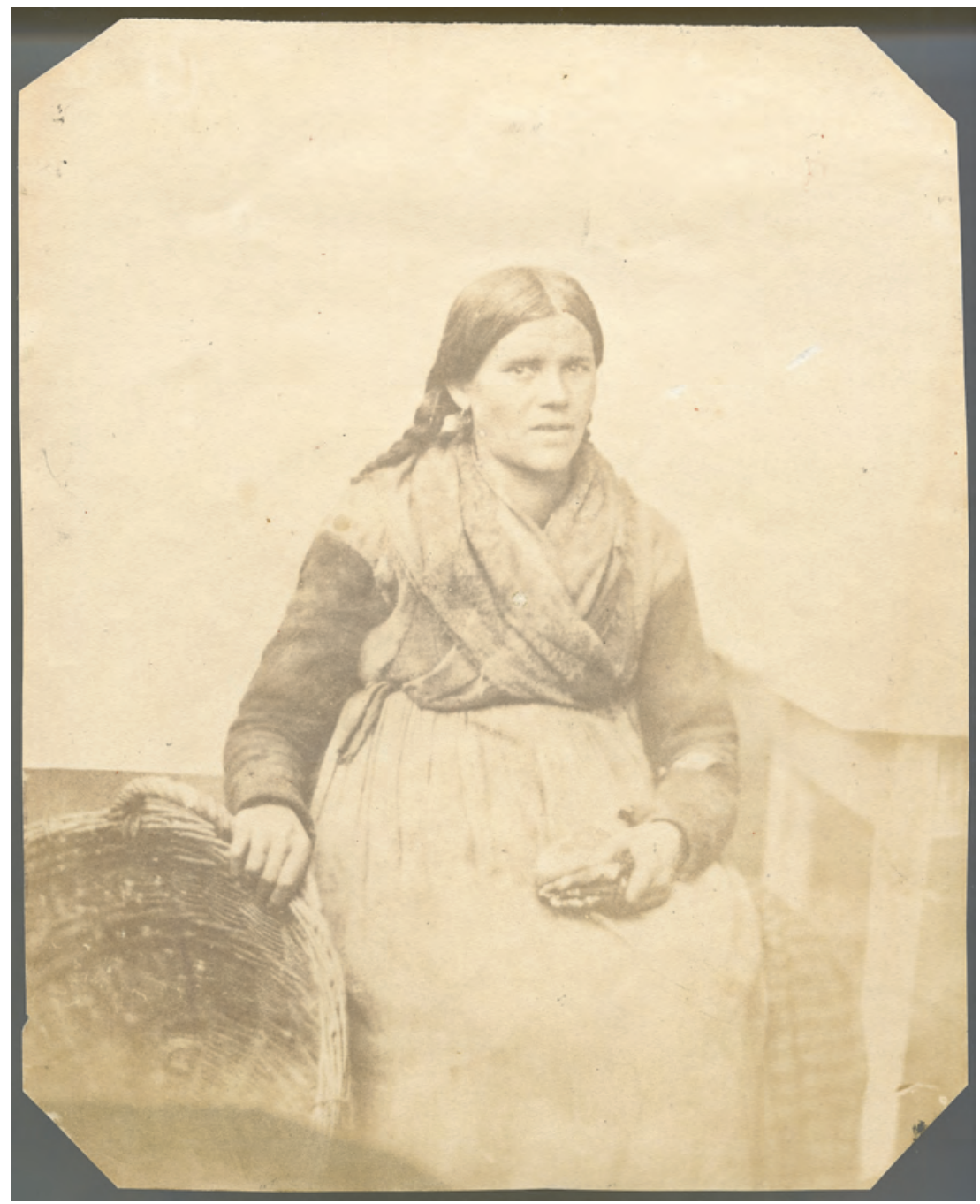

Abb. 4: Carl Durheim, Axt, Magdalena [35 Jahre alt, Beibälterin des Lorenz Pfaus (falsch Christian Manz) aus Tautmergen, Württemberg], 1852/53, Fotografie, Salzpapierabzug, $14 \times 17 \mathrm{~cm}$ (Schweizerisches Bundesarchiv, Bern, CH-BAR 30313821; Wikimedia Commons)

Die Bilder der „Heimatlosen“ entstanden in einer Zeit, in der die Fotografie als Medium neu aufkam und im erkennungsdienstlichen Kontext langsam begann, die schriftliche Personenbeschreibung und gezeichnete Bild- 
nisse zu ergänzen oder zu ersetzen. ${ }^{18}$ In ihrer Funktion zur Wiedererkennung ersetzten sie auch die körperliche Brandmarkung, die in den meisten Ländern Europas in der ersten Hälfte des 19. Jahrhunderts verboten worden war. ${ }^{19}$ Thomas Meier und Rolf Wolfensberger haben die Unsicherheiten und Überlegungen der beteiligten Akteure anhand der Korrespondenz zwischen dem Fotografen Durheim, dem Lithografen, dem zuständigen Bundesanwalt, der Justiz und dem Bundesrat für die Schweizer Heimatlosenfotografie herausgearbeitet (vgl. Meier/Wolfensberger 1998a, S. 11-20). Auch anhand der Fotografien selbst lässt sich das anfängliche Experimentieren verdeutlichen. Ein einheitliches Verfahren, wie die bis heute bekannten „Verbrecherbilder“ oder mug shots, war noch nicht etabliert. Unter den für diesen Beitrag ausgewählten Fotografien aus der Sammlung ist das Bild von Barbara Brutschi (Abb. 3) vermutlich das ältere. Aus den Korrespondenzen ist bekannt, dass Durheim bei den ersten Fotografien der "Heimatlosen“ nur den Oberkörper erfasste. Dies kam ihm jedoch nach kurzer Zeit als ein zu geringer Ausschnitt für die Wiedererkennung vor, weshalb er in späteren Bildern versuchte, möglichst den gesamten Körper abzulichten (vgl. ebd., 14, 16).

Mitte des 19. Jahrhunderts war eine fotografische Bildsprache noch kaum entwickelt. Susanne Regener (1999, S. 27-63) hat in ihrer groß angelegten Studie die anfängliche Ähnlichkeit von Bürger- und (vermeintlichen) Verbrecherporträts im globalen Kontext nachgewiesen. Die Ähnlichkeit lag unter anderem darin begründet, dass die Polizeifotografien von professionellen Atelierfotografen wie Durheim, die ihre Berufserfahrung mit bürgerlichen Auftraggeber_innen gesammelt hatten, hergestellt wurden. So waren die dargestellten Gefangenen meist neben einem kleinen Tisch platziert. Einige von ihnen wurden nachweislich in fremden Kleidern abgelichtet, und in manchen Bildern wurde qua Attribut der Beruf gekennzeichnet, so zum Beispiel in dem Bildnis der Korbflechterin Magdalena Axt (Abb. 4). Auch bürgerliche Requisiten wie Schirmmützen und Bücher kamen ungeachtet dessen, dass die Abgelichteten wohl kaum des Lesens mächtig waren, zum Einsatz (vgl. Meier/Wolfensberger 1998a,

18 Zur Geschichte des vorfotografischen Verbrecherbildnisses im Porträtformat vgl. Jeutter 2016.

19 Auf dem Gebiet der heutigen Schweiz wurden Leibesstrafen wie Brandmarkung, Galgen, Rad und körperliche Züchtigung durch das „Peinliche Gesetzbuch der helvetischen Republik“ im Jahr 1799 nach Einmarsch französischer Truppen abgeschafft und durch Freiheitsstrafen abgelöst. Für eine sozialphilosophische Deutung und Analyse der Abschaffung körperlicher Strafen mit dem Ende des Ancien Régime vgl. Foucault 1989, S. 9-25. 
S. 16). ${ }^{20}$ Aufgrund der langen Belichtungszeit wurden, wie auch bei der bürgerlichen Atelierfotografie, Kopfstützen eingesetzt, die teilweise noch auf den Bildern erkennbar sind, zumeist aber retuschiert wurden (vgl. ebd., S. 15). Ebenso wurde der Hintergrund der Bilder herausgeschnitten, da er als unwichtig oder sogar ablenkend wahrgenommen wurde und die Arbeit des Lithografen nicht stören sollte (vgl. Gasser 1998, S. 138). Nur anhand weniger schlecht oder nicht retuschierter Abzüge lässt sich nachvollziehen, dass die meisten Fotografien im Innenhof des Gefängnisses und einige wenige im Fotoatelier von Durheim aufgenommen wurden.

Obwohl die Bilder retuschiert und die Dargestellten mit Requisiten ausgestattet wurden, mangelte es zu dieser Zeit an Bewusstsein dafür, dass jede Fotografie eine Konstruktionsleistung ist. Die ersten Fotografen wie Louis Jacques Mandé Daguerre und William Henry Fox Talbot beschrieben in den 1830er und 1840er Jahren das Fotografieren als „Abzeichnen“ oder "Selbsteinschreibung“ der Natur ins Bild (Leimgruber 2005, S. 215). Im Gegensatz zur Malerei empfanden sie die Fotografie als sehr viel weniger manipulierbar. Man könne beispielsweise die Tageszeit am Lichteinfall erkennen und unschöne Details im Hintergrund nur bedingt verdecken (vgl. Geimer 2010, S. 60-63, 113-116 und 171-173). Dies führte zu einer weitgehenden Blindheit gegenüber der Tatsache, dass jede Fotografie doch nur einen Ausschnitt der Realität zu einem ganz bestimmten Zeitpunkt und aus einem bestimmten Blickwinkel festhalten kann.

Der Glaube an die Objektivität der Fotografie war in der Mitte des 19. Jahrhundert sehr verbreitet, und bis heute wirken Fotos und Filmaufnahmen objektiver und realer als gemalte Bilder. Susan Sontag zeigt die Dialektik von Bild und Wirklichkeit in der modernen Gesellschaft daran auf, dass es mittlerweile sogar zu einer Verkehrung gekommen ist: Nicht Fotografien werden für etwas Reales gehalten, sondern die Wirklichkeit wirkt „mehr und mehr so, wie das, was uns die Kameras zeigen.“ (Sontag 1987, S. 153-154). Dadurch würde, so Sontag, einerseits dem Bedürfnis nach Vereinfachung der komplexen gesellschaftlichen Verhältnisse im Kapitalismus nachgegangen (vgl. ebd., S. 154). Andererseits diene die Fotografie auch dazu, den fotografierten Gegenstand unter Kontrolle zu bringen, indem er klassifizierbar werde (vgl. ebd., S. 148). Mitte des 19. Jahrhundert war die Bedeutung, die die Fotografie für die moderne Gesellschaft erlangen sollte, jedoch noch nicht offensichtlich. Wie beschrieben, wurden ver-

20 Dies bedeutet keinesfalls, dass es nicht auch bürgerliche Existenzen unter Sinti_ze, Rom_nja und Jenischen gab. Bei den Abgelichteten handelte es sich in diesem Zusammenhang aber ausschließlich um „Heimatlose“. 
schiedene Verfahren tastend erprobt und entwickelt; die (überwiegend männlichen) Fotografen und ihre Auftraggeber_innen changierten dabei zwischen unkommentiertem Retuschieren und unreflektiertem Glauben an eine vermeintliche Objektivität des Bildes.

Das Experimentieren beschränkte sich allerdings nicht nur auf die technische Seite der Fotografie, sondern betraf auch die gesellschaftspolitische Wirkweise des Mediums. Die meisten der erkennungsdienstlich zu erfassenden Personen waren mutmaßlich zum ersten Mal mit einem Fotoapparat konfrontiert. Zudem unterschied sich das durch Zwang geprägte Setting vom freiwilligen Besuch im Atelier eines Fotografen. Während das primäre Ziel des Einsatzes von erkennungsdienstlichen Bildern das leichtere Wiedererkennen der erfassten Personen war, wurde von staatlicher Seite zudem zufrieden festgestellt, dass die Fotografien einschüchternd wirkten. Dies betraf sowohl die Wirkung der Bilder als auch den Aufnahmeprozess. ${ }^{21}$ Die Betroffenen selbst schilderten den Prozess des Fotografiert-Werdens als furchteinflößend. So sagte eine im Oktober 1852 fotografierte Frau laut Verhörprotokoll folgendes aus: „Dann muss ich sagen, als man mein Porträt genommen, dass ich glaubte, man wolle mich totschiessen. Ich wusste nicht, was in der Maschine ist. (...) O! Angst habe ich gehabt! Ich dachte bei mir selbst, als ich auf dem Stuhl g'hocket bin: ich bin dem Herrgott doch nur einen Tod schuldig!" (zit. nach Meier/Wolfensberger 1998a, S. 16). Solche Zeugnisse von Betroffenen sind selten überliefert und auch in diesem Fall nur aus zweiter Hand, protokolliert durch einen Schweizer Beamten. Sie geben wertvolle Einblicke in die Perspektive der Betroffenen. Die zitierte Aussage zeigt mehrere Dimensionen der Versicherheitlichung auf und weist gleichzeitig auf eine Ambiguität von Sicherheit im Kontext von Differenz. Die Sicherheit, die für die staatliche Seite und vermeintlich für die gesamte Gesellschaft durch die voranschreitende Überwachung und Einschüchterung hergestellt werden sollte, steht dem Verlust des Gefühls von Sicherheit auf der Seite der versicherheitlichten „Heimatlosen“ entgegen.

Vergleichbar mit den Hinrichtungsprozessen, die noch im 18. Jahrhundert als Spektakel inszeniert wurden, waren auch die frühen Polizeifotografien dafür geeignet, sich an ein breiteres Publikum zu richten und somit für eine massenmediale Versicherheitlichung eingesetzt zu werden. Bereits die Fotografien der „Heimatlosen“ in der Schweiz wurden in Form

21 Im Schweizerischen Bundesblatt 1853 wurde beispielsweise resümiert, dass die Aufnahmeprozedur bei den Betroffenen als „moralisches Schreckmittel“ (zit. nach Meier/Wolfensberger 1998a, S. 15) gewirkt hätte. 
von Lithografien in Alben zusammengestellt und vervielfältigt. Darauf folgten erste druckgraphisch reproduzierte Fotografien von Kriminellen oder für kriminell Gehaltenen in Fahndungsblättern, die jedoch mit zumeist ein bis zwei Abbildungen pro Jahr in der Zeit von 1853 bis 1870 noch eine Seltenheit waren (vgl. Regener 1999, S. 94). In den 1870er Jahren kam es zur Herstellung von sogenannten „Verbrecheralben“, in denen Fotografien der Gesuchten gesammelt waren (Jäger 2009, S.375). Auch hier wurde noch experimentiert, so wurde beispielsweise versucht, mittels Spiegeln mehrere Seiten des Kopfes in einer Fotografie abzulichten (vgl. Meier/Wolfensberger 1998a, S. 20; Regener 1999, S. 111-112). Wenngleich die Alben zunächst für den polizeiinternen Gebrauch bestimmt waren, wurden sie bald auch Zeug_innen vorgelegt, um Täter_innen zu identifizieren. Die öffentlich einsehbaren Fahndungsbilder hatten einen einschüchternden Effekt auf die gesamte Bevölkerung, die der Stigmatisierung durch Ablichtung entgehen wollte, und stellten somit ein historisch machtvolles Medium visueller Versicherheitlichung dar.

\section{Standardisierung der erkennungsdienstlichen Polizeifotografie am Beispiel Bayerns um 1900}

Auf die Zeit der experimentellen Anfänge, während der auch immer wieder grundsätzlich an der Eignung von Fotografie für den polizeilichen Einsatz gezweifelt wurde, folgte eine Phase der Standardisierung des Aufnahmeverfahrens und seiner Ästhetik. Für Sinti_ze und Rom_nja bedeutete die Darstellung nach Art einer zunehmend etablierten Form der ermittlungsdienstlichen Fotografie die visuelle Gleichstellung mit Serienstraftäter_innen. Die polizeilich eingesetzte Fotografie führte somit zu einer neuen Dimension der visuellen Versicherheitlichung, die eine weitere Kriminalisierung und einen drohenden oder tatsächlichen gesellschaftlichen Ausschluss zur Folge hatte.

Eine der wichtigsten Personen in der Weiterentwicklung der polizeilichen Fahndungsfotografie, der französische Kriminalist Alphonse Bertillon (1853-1914), stand der Fotografie selbst lange Zeit skeptisch gegenüber. $^{22}$ 1879/80 entwickelte er ein umfassendes System zur Personenidentifizierung, welches hauptsächlich auf der Vermessung und Beschreibung der betroffenen Personen inklusive zahlreicher Körperteile beruhte, deren

22 Bertillon empfand die Deutung von Fotografien als zu subjektiv und bevorzugte quantifizierbare Zahlen. Vvgl. Kammerer 2007, S. 34. 
detaillierte Ergebnisse in Karteikarten vermerkt wurden (vgl. Regener 1999, S. 131-146). Dabei kamen eigens entwickelte Messgeräte, etwa für die Vermessung des rechten Ohrs, zum Einsatz. ${ }^{23}$ Sie sollten die Vergleichbarkeit über die einzelnen Polizeistationen hinaus gewährleisten.

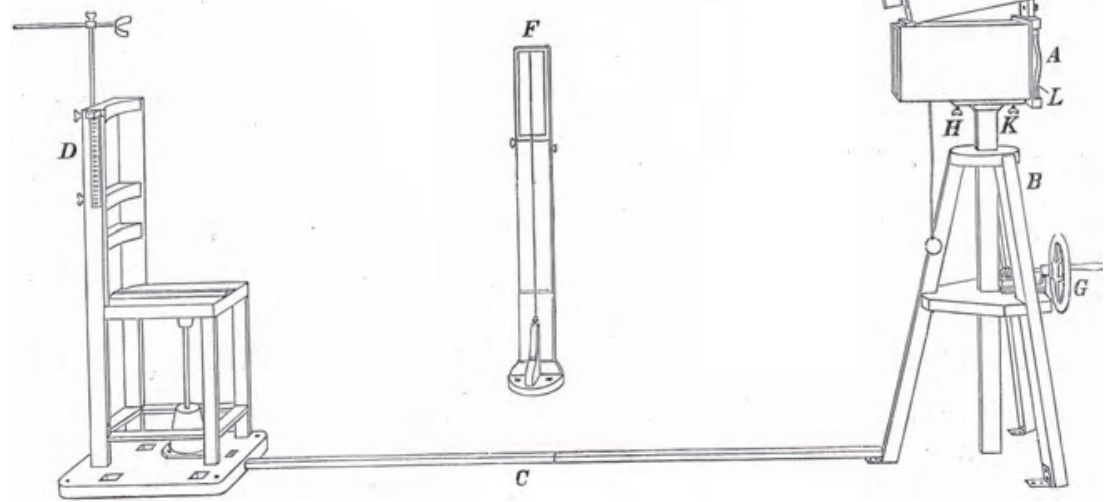

Abb. 5: Aufnahmeapparatur für die erkennungsdienstliche Fotografie nach Bertillon, aus: Anweisung zur Vornabme von Körpermessungen und Beschreibungen nach dem System von Alphonse Bertillon, herausgegeben von Eduard von Meerscheidt-Hüllesem, Berlin 1899, S. 61

Das Identifikationssystem beinhaltete darüber hinaus eine Methode zur fotografischen Erfassung der Personen, welche in den darauffolgenden Jahrzehnten auch im Kontext der polizeilichen Fotografie von Sinti_ze und Rom_nja verwendet wurde. Bertillon hatte dazu eine Apparatur entwickelt, die aus einem drehbaren Stuhl mit Kopfstütze und einer in genauem Abstand befestigten Kamera bestand (Abb. 5). Mit ihr konnten zwei Fotografien aufgenommen werden, die später nebeneinander auf einem Träger zu sehen waren. Bertillon empfahl die Kombination einer en face mit einer Profilaufnahme, wie sie bereits in den 1860er und 1870er Jahren für die Personendokumentation in der Anthropologie verwendet wurde (vgl. Jäger 2006, S. 205). Dabei wurde auf das Format „Brustbild“ zurückgegriffen, welches der Fotograf der Schweizer Heimatlosenbilder Durheim Mitte des 19. Jahrhunderts noch als zu ungenau empfunden hatte. Bertillon legte von der Größe der Bilder bis zur einheitlichen Auswahl der Seite

23 Ein Bild des Ohrmessers nach Bertillon findet sich bei Vec 2002, S. 46. 
des Profils (rechts) alles exakt fest, um die Vergleichbarkeit der Fotografien sicherzustellen. Bis heute ist die Fotografie das Element der Bertillonage, welches die Verfahren des polizeilichen Ermittlungsdienstes am nachhaltigsten geprägt hat. Die aufwendigen Vermessungen wurden über die Zeit weitgehend durch Fingerabdruckverfahren abgelöst.

Anhand der Entwicklung der Polizeifotografien von Sinti_ze und Rom_nja in Bayern zwischen 1899 und 1937 lässt sich die zunehmende Standardisierung nachweisen. Bayern eignet sich besonders als Beispiel, da es mit der Einrichtung eines „Nachrichtendienst für die Sicherheitspolizei in Bezug auf Zigeuner“ (kurz „Zigeunerzentrale“) in München 1899 über die früheste und größte polizeiliche Datensammlung auf diesem Feld verfügt. Sie wurde mit dem Ziel angelegt, alle in Bayern aufgegriffenen „Zigeuner" und diejenigen, „welche nach Zigeunerart umherziehen“ (Dillmann 1905, S. 9), zu erfassen. Die Zentrale operierte mit modernen Technologien wie Fotografie, Fingerabdrücken, Telegrafendienst und Kennkarten (vgl. Heuß 2003, S. 24), welche in einem Karteisystem angelegt wurden. Die Einrichtung der spezialisierten Polizeistelle geschah infolge einer Verschärfung der "Zigeuner"-Gesetzgebung seit 1885 in Bayern, Preußen und anderen deutschen Staaten (vgl. Opfermann 2016, S. 62).

Diese Art der Überwachung war nicht nur neu in Bezug auf die Größe und die eingesetzte Technologie. Ungewöhnlich war auch, dass die Daten aufgrund einer Gruppenzugehörigkeit und nicht aufgrund tatsächlicher Straftaten gesammelt wurden. Zeitgenössische Debatten in den Lokalzeitungen zeigen, dass diese Praxis durchaus kritisch aufgenommen wurde. So schrieb etwa Adolph Weckel 1902: “... jedes Strafgesetz setzt eine strafbare Handlung voraus, daß aber die bloße Existenz eines Volks eine strafbare Handlung sei, war für diese alten Richter etwas neues." (Welcker 4./5.2.1902, S. 243)..$^{24}$

Reproduktionen der ältesten Fotografien aus der Münchener Zentrale finden sich heute in einem Buch, welches 1905 vom Chef der Bayerischen Polizei Alfred Dillmann unter dem Namen „Zigeuner-Buch" veröffentlicht wurde. Es erschien in hoher Auflage mit 7.000 Exemplaren und wurde an Polizeistellen im In- und Ausland verteilt. Die Porträt-Fotografien sind im Kontext des Buches und der „Zigeunerzentrale“ zu verstehen. Das Buch beginnt mit einer Einleitung von Dillmann über die angenommene Geschichte der "Zigeuner“ in Europa, über das angebliche Problem der Kriminalität von „Zigeunern“ und über die aktuelle Gesetzeslage. Damit greift es das Schema des 178 Jahre zuvor erschienenen Buchs von Weissen-

24 Vgl. auch Heuß 2003, S. 23. 
bruch wieder auf. Dillmann unterscheidet zwischen inländischen und ausländischen „Zigeunern“ und betont, dass es kaum noch „raßechte Zigeuner" (Dillmann 1905, S. 5) gebe, da eine Vermischung mit der einheimischen Bevölkerung stattgefunden habe. ${ }^{25}$ Die Notwendigkeit einer umfassenden Überwachung rechtfertigt er damit, dass die „Zigeunerplage [...] die öffentliche Sicherheit schwer gefährdet“ (ebd., S. 6).

Der Hauptteil des Buchs besteht aus einer Liste von 3.350 Namen, die teilweise mit kurzen Personenbeschreibungen versehen sind. Die Beschreibungen betreffen Religion, Herkunft, Familiennetzwerke, die Arbeit, das Aussehen und - falls vorhanden - begangene Verbrechen und Strafen. Darauf folgt eine Tabelle mit äußerlichen Besonderheiten oder Kennzeichen einzelner Personen, etwa auffällige Narben, Tätowierungen oder verkrümmte Finger. Am Ende des Buches befinden sich 32 Fotografien von Personen, die im Hauptteil verbal beschrieben werden. Es lässt sich nicht pauschal feststellen, dass die gezeigten Personen besonders schwere Straftaten begangen hätten. In der Einleitung steht lediglich, dass es sich um „eine Auswahl bemerkenswerter Zigeuner-Fotographien“ (Dillmann 1905, S.9) handle, die der Hauptsammlung der Fotografien in der Münchener "Zigeuner-Zentrale" (ebd., S.9) entnommen seien. ${ }^{26}$ Weshalb die abgedruckten Fotografien bemerkenswert sind, wird nicht weiter spezifiziert. Da die Fotografie um 1900 noch ein teures Verfahren war, lässt sich festhalten, dass die Dargestellten für so bildwürdig gehalten wurden, dass der Staat bereit war, ihre fotografische Erfassung zu finanzieren. Die Datensammlungen zu "Zigeunern" können folglich als Zeichen dafür gelesen werden, welche Wichtigkeit dem Unterfangen von staatlicher Seite beigemessen wurde.

Die Ablichtungen am Ende des Buchs bestehen aus Einzelportraits, zumeist Bruststücken, teilweise sind die Personen aber auch stehend in ganzer Figur oder mit dem Oberkörper abgelichtet. Eines der Bilder wurde mit dem oben beschriebenen Spiegelverfahren aufgenommen (vgl. Dillmann 1905, S. 335). Die Vielfältigkeit der Aufnahmen zeigt, dass auch zu

25 Unterscheidungen dieser Art haben sich bis in die NS-Zeit gehalten, in der sie dem Leiter der Rassenhygienischen und bevölkerungsbiologischen Forschungsstelle (RHF) Robert Ritter dazu dienten, gegen sogenannte „Zigeunermischlinge“ noch härter vorzugehen als gegenüber denjenigen, die in der Rassenlogik des NS zumindest als reines Volk gelten konnten. Vgl. Luchterhandt 2007, S. 326.

26 Einzelne weitere Personenbeiträge sind mit dem Zusatz versehen, dass sich Fotografien in der Sammlung der Zentrale befänden, was bestätigt, dass die eigentliche Sammlung größer ist und es sich tatsächlich um eine Auswahl handelte, vgl. etwa den Eintrag zu Maria Krems in Dillmann 1905, S. 127). 
diesem Zeitpunkt noch kein einheitliches Verfahren verbreitet war. Exemplarisch soll hier das Bild der Viktoria Wagner genauer betrachtet werden (Abb. 6). Ihr Bild ist ein typisches Hüftbild. Sie schaut frontal in die Kamera, ihre Arme sind leicht angewinkelt und die Hände sind gut sichtbar auf Hüfthöhe platziert. ${ }^{27}$ Der textliche Eintrag zu ihrer Person verweist darauf, dass sie bereits wegen Diebstahl, Gaukelei, Bettelei und Landstreicherei bestraft wurde (Abb. 7). Hier findet eine andere Form der Verbindung von

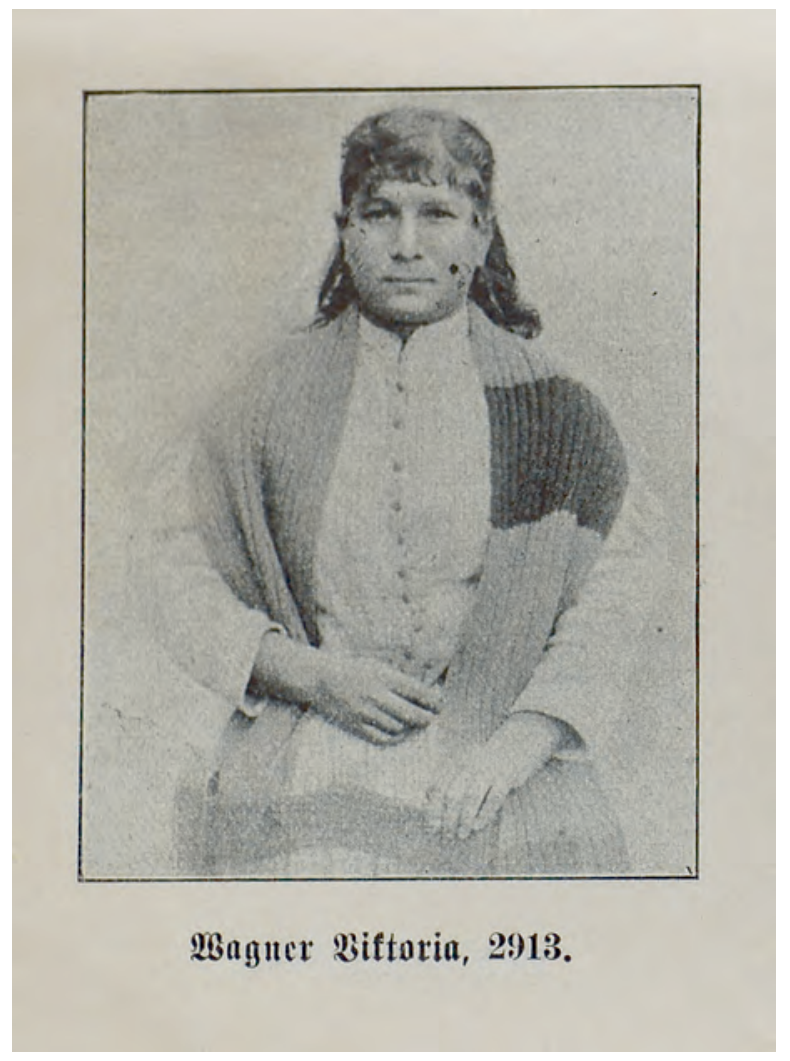

Abb. 6: Fotografie von Wagner, Victoria, aus: Dillmann 1905, S. 341

27 Vgl. Regener (1999, S. 112, 243-244) zur Rolle der Hände in der ermittlungsdienstlichen Fotografie um 1900. 
2909. Wagner Rofa, geb. ungef. 1895, f. Wagner நer= Łules, $\Re$ x. 2866.

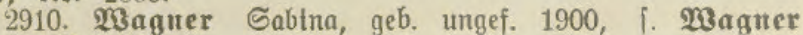
Auguit, $\mathfrak{R r} .2842$.

2911. Mzaguer Sophic, geb. ungef. 1890, †. Midjter Mtulibald, $\mathfrak{N}$ r. 2140.

2912. 2Kaguer Balentin, geb. 28.11.1888, โ. Waguer

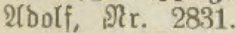

2913. $\mathfrak{B a g n e r} \mathfrak{B}$ iftoria Magbalena (angebl.), nennt fich nudi) Miettbach u. Sattelt, bermutl. leb., Bigeunerin, Sdjirm= madjerin, Beb.= Beit u. = Drt, Relig., 2rbítammung, Seimat u.

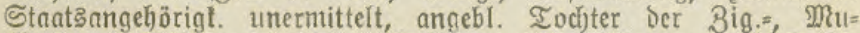
fiters= u. Edjirmmacheršehel. Jo ohann u. (5va Bangner, geb. Delis, alias Tochter bon Szeinrich u. Ehriftine Wagner, geb. Etein= bach, angebl. berljeir. nit bem Bigeuner, Mufiffer u. Sammer= jäger Johann Mettbach) (f. Diefen), beh)auptet, im গ̃. 1875 alias 1880 in Benf, alias in St. (Sallen, 厄cirrz., ceb. u. in Mem=

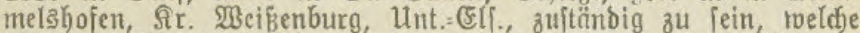
2ngaben fich als folf fh erroicfen haben, zieht mit einem 3igeuner

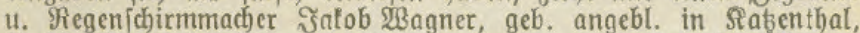

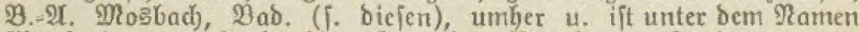
Mettbach) reg. Dicbitahls, (Sauleclei, Bettele u. Sanditr., unter

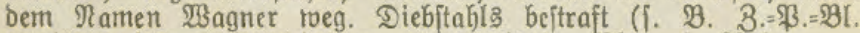
154/10521/03, 38/2222/04 u. 41/2382/04). \$hot. i. 2Unh.

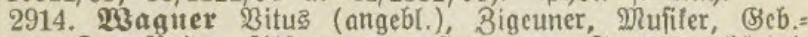
Beit u. = Drt, Relig., श̂kitammung, Seimat u. Staatకangehörigt. uncrmittelt, behauptet fäl(ç)l., am 5.12.1878 in Sagenau, $\Re .=\Phi$. onf., Unt.=Elf., geb. u. in Sagenau zuitändig zu jein, angebl. verbeir. mit einer $\mathfrak{F} \mathrm{r}$ i b a $\mathfrak{B}$ a gne $\mathrm{r}$ ( $\{$. bieje), twurbe am 3.10.1904 in ber Nähe bon Sberbaufen, $\mathfrak{B} .=\mathscr{2}$. Sa fienfurt, in

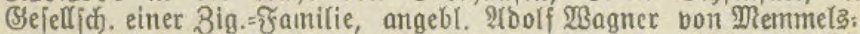
lojen, $\Re .=$ D. WBeibenburg, Itnt.=Eli., angetroffen. Die angebl.

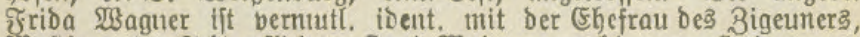
Mnifiters u. Sthimftiders Sarl Maier, angebl. bon Şolzen, pr. Pieg. $=$ Bez. 2rabiberg (i. biefen).

2915. 23aguter Withclmine, geb. Reinljarbt, †. 23agner Mickael, $\Re$ r. 2898.

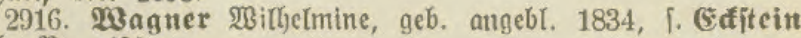
Sarl, গr. 492 .

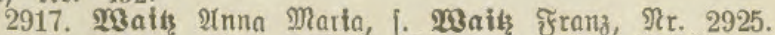

2918. Waath Ninton, fntt?., Bigeuner, Bferdebändter bon

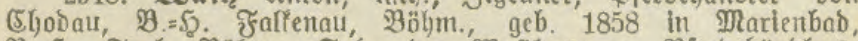

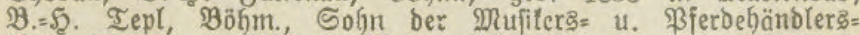

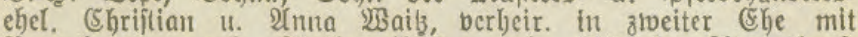
$\Re$ a rbara, geb. Stoifa (tath., geb. 1872 in 2 bzgersoborf, $\mathfrak{B} .=5$. Shtebing, Mieb.=Deft., ill. ber Rigeunerin Therefe Stoifa), zog bisher mit peinten Brübern Sart u. (Emil $\mathfrak{B a i b}$ u. Der Banbe Beorg Babba unker u. Katte im Mai 19038 angebr. nus ber eriten (5he mit Magdalena, geb. Berger, herborgegan. gene Situber bei fich), Deren \$rerionalien er, twie folgt, angab:

Abb. 7: Buchseite mit Eintrag zu Wagner, Viktoria, aus: Dillmann 1905, S. 285 
Delinquenz und Bild statt. Das Vergehen und der Strafvollzug werden nicht mehr direkt im Bild dargestellt, wie dies bei „Zigeunerwarntafeln“ oder Hinrichtungsbildern der Fall war, sondern durch Text und Bild auf verschiedene Abschnitte des Buches verteilt und dennoch durch die Unmissverständlichkeit der Worte und die Logik des publizistischen Mediums miteinander verbunden. Dies kann als neue Verschränkung von Bildund Textmacht gedeutet werden, durch die abweichendes Verhalten mit den abgedruckten Bildern und dem Aussehen zunächst einzelner Personen verknüptt wird. Im Kontext eines Buches, das als „Zigeuner-Buch“ veröffentlicht wurde, und der einhergehenden praktischen Polizeiarbeit wird die gesamte Gruppe, die als "Zigeuner“ imaginiert wird, als Gegenbild zu den nicht-kriminellen, zivilisierten Deutschen oder Bayern genutzt. Dazu werden fotografische und insofern glaubwürdige Abbildungen von einzelnen Personen genutzt.

Anhand der frühen Fotografien aus Bayern lässt sich die Entwicklung zum standardisierten Verbrecherbild nachvollziehen. Die FotografieSammlung der "Zigeunerzentrale“ aus München wurde gemeinsam mit den bis dato erstellten Personenakten 1919 während der Räterepublik durch die Kommunisten verbrannt, wie aus verschiedenen Zeitungsartikeln der Zeit hervorgeht. ${ }^{28}$ Einzelne frühe Bilder aus Bayern sind jedoch vermutlich in anderen Polizeistationen erhalten geblieben. Sie wurden gemeinsam mit einer neu angelegten Sammlung aus München in der Zeit des Nationalsozialismus nach Berlin gebracht. Zum Zeitpunkt des Umzugs 1938 bestand die Sammlung der Bayerischen "Zigeunerzentrale“ aus 17.951 Akten..$^{29}$ In Berlin wurden ab 1938 verschiedene lokale Sammlungen aus ganz Deutschland zusammengeführt und von der sogenannten „Rassenhygienischen und bevölkerungsbiologischen Forschungsstelle“ (RHF) des NS-Gesundheitsministeriums verwendet (vgl. Fings 2008).

28 Vgl. Zeitungsberichte (StadAM ZA-13241).

29 Diese Zahl stammt aus einer Auflistung der Zigeunerpolizeistelle München vom 13.10.1938, in der es heißt, dass 16.104 Akten an Berlin übergeben wurden und 1.847 Akten in München verblieben sind (StAM 1546, Polizeidirektion München 7033). 

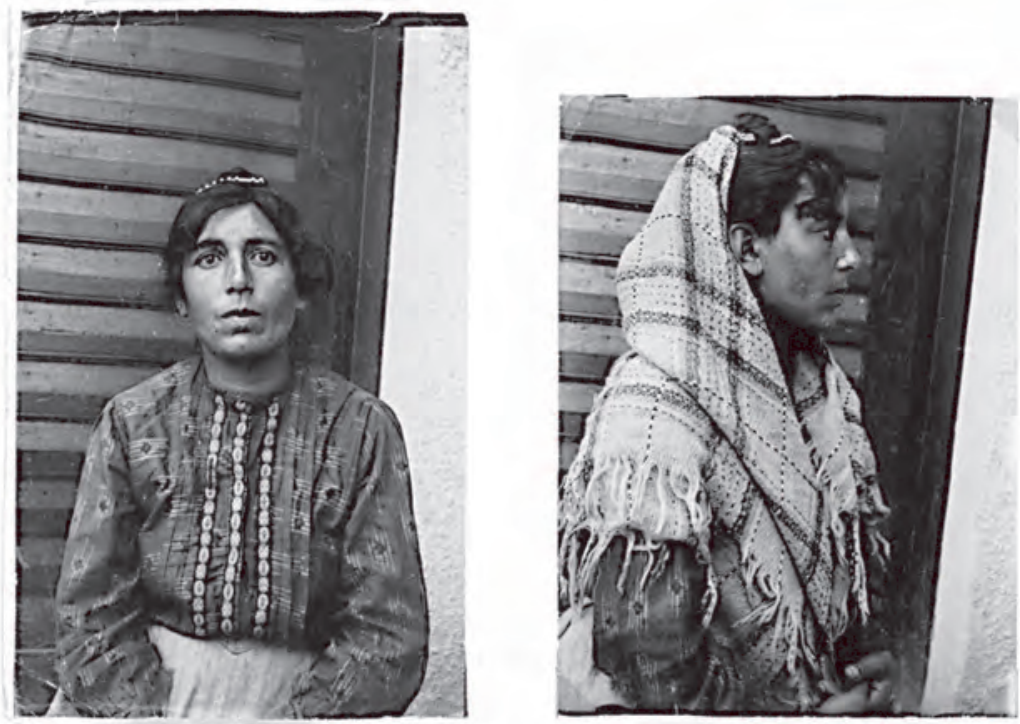

Abb. 8: Pauline Reinhardt, Oktober 1911 in Kempten, 2 Fotografien (BArch R-165-57)

Pouline Reimberde $\times 20.12 .1881$.
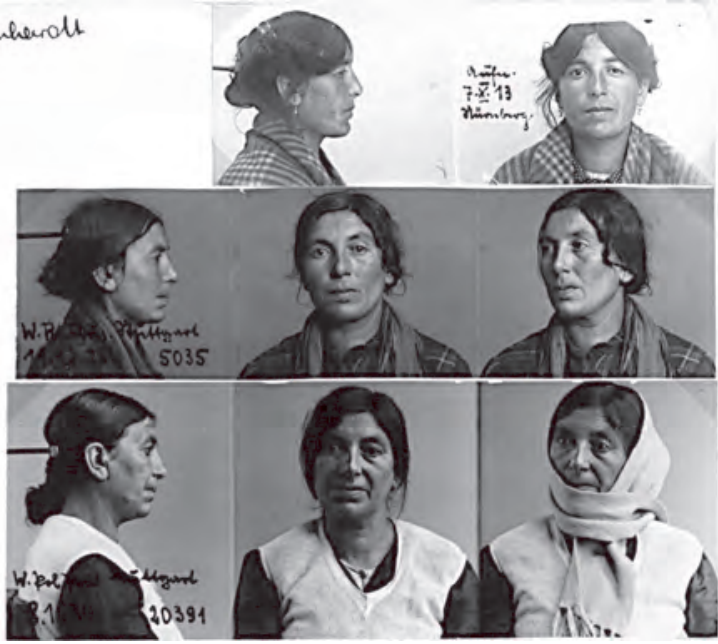

Abb. 9: Pauline Reinhardt, geb. 20. Dezember 1881; oben: 7. Oktober 1913 in Nürnberg; Mitte: 11. Dezember 1925 in Stuttgart; unten: 10. Oktober 1937 in Stuttgart (BArch R-165-57) 


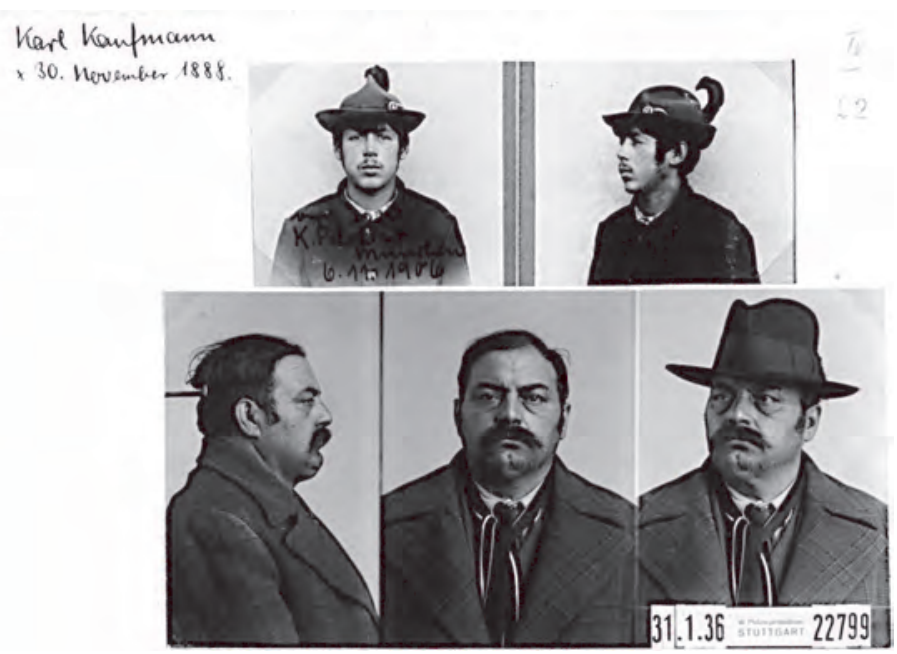

Abb. 10: Karl Kaufmann, geb. 30. November 1888; oben: K. Pol. Dir. München, 6. November 1906; unten: W. Polizeipräsidium Stuttgart, 31. Januar 1936 (BArch R-165-52)

Die Entwicklung der Fotografiestandards lässt sich besonders gut anhand von Bilderreihen nachvollziehen, bei denen eine Person über mehrere Jahrzehnte immer wieder von der Polizei abgelichtet wurde. Zwei solcher Reihen sollen hier exemplarisch analysiert werden. Zunächst bestätigen die wiederholten Aufnahmen, dass als "Zigeuner" oder "Zigeunerin“ stigmatisierte Personen ihr Leben lang immer wieder mit der Polizei konfrontiert waren und der Prozedur der Fotografie unterzogen wurden. Die Bilderreihe von Pauline Reinhardt, geboren am 20. Dezember 1881 (Abb. 8 und 9), belegt, dass sie mindestens 1911, 1913, 1925 und 1937 von der Polizei aufgegriffen wurde. Die erhaltenen Fotografien von Karl Kaufmann, geboren am 30. November 1888 (Abb. 10), bestehen aus einer Bilderreihe aus München von 1906 und einer Bilderreihe aus Stuttgart von 1936. Der Erhalt der Münchener Bilder spricht in Anbetracht der Zerstörung der gesamten Sammlung in der Münchener Räterepublik dafür, dass einige Bilder der Zentrale an andere Polizeistationen weitergegeben wurden. ${ }^{30}$

30 Es lässt sich vermuten, dass die Münchener Fotografien zumindest teilweise als Duplikate nach Stuttgart gegeben wurden, nachdem seit 1903 ein Überein- 
Die ältesten Fotografien von Karl Kaufmann aus dem Jahre 1906 bestehen im Gegensatz zu den Bildern aus dem "Zigeuner-Buch“ aus zwei Aufnahmen (Abb. 10 oben). Sie entsprechen in mehrerlei Hinsicht noch nicht dem Aufnahmeverfahren der Bertillonage: Während das erste Bild in Frontalansicht aufgenommen wurde, handelt es sich beim zweiten Bild um eine Dreiviertelansicht von links. Die Bertillonage hätte hier ein Profilbild von der rechten Gesichtshälfte verlangt. Zudem lässt die fehlende Kopfstütze darauf schließen, dass die Fotografien ohne Bertillons Aufnahmeapparatur gemacht wurden. Auch bei den ältesten vorhandenen Bildern von Pauline Reinhardt, die immerhin fünf Jahre später im Jahre 1911 in Kempten aufgenommen wurden (Abb. 8), wurde noch keine Aufnahmeapparatur eingesetzt. Sie sind eindeutig im Freien vor einer Hauswand aufgenommen worden. Die Reihe besteht zwar aus Frontalansicht und Profilbild, jedoch weicht das Format mit einer Ansicht bis zur Hüfte von den sich entwickelnden Standards ab. Auf dem Profilbild ist ein weiteres Detail zu erkennen, welches sich in den folgenden Jahrzehnten etablieren sollte: In der Profilaufnahme ist Pauline Reinhardt mit einem Kopftuch zu sehen, welches sie in der Frontalansicht nicht trägt. Vermutlich erhofften sich die Ermittler, durch die unterschiedlichen Inszenierungen die Wiedererkennbarkeit zu erhöhen.

1913 wurden in Nürnberg die ersten Studioaufnahmen von Pauline Reinhardt gemacht (Abb. 9, obere Reihe). Auch sie bestehen aus Frontalund Profilbild, zeigen im Vergleich zu den vorherigen Fotografien aber eine Metallstütze zur Festlegung der Position des Kopfes und einen neutralen Hintergrund. Vermutlich wurden diese Bilder bereits nach dem Verfahren der Bertillonage angefertigt. Auf der nächsten Fotoreihe (Abb. 9, mittlere Reihe) von 1925 aus dem Württembergischen Polizeipräsidium Stuttgart lässt sich eine weitere Angleichung an die sich auch international durchsetzenden Standards erkennen. So kommt ein drittes Bild hinzu, welches eine Dreiviertelansicht von links ergänzt. Diese Dreier-Reihen werden zur typischen Polizeibild-Reihe. Auf den Fotoreihen von Karl Kaufmann und Pauline Reinhardt, die 1936 (Abb. 10, unten) und 1937 (Abb. 9, unten) in Stuttgart aufgenommen wurden, wird an der DreierReihe festgehalten. Karl Kaufmann trägt auf dem letzten Bild einen Hut, Pauline Reinhardt ein Kopftuch. ${ }^{31}$ Laut der Sortierung, die in der NS-Zeit

kommen zwischen Bayern und Württemberg zum Nachrichtenaustausch auf Ebene der Sicherheitspolizei in Bezug auf das "Zigeuner"-Thema bestand (BArch R1501-113700, 67), und somit der Vernichtung der Akten 1919 entgangen sind.

31 In der Akte von Pauline Reinhardt befinden sich zudem noch zwei undatierte Bilder, die dem Inhalt nach Anfang der 1930er Jahre aufgenommen wurden 
durch die RHF vorgenommen wurde und an der sich die archivalischen Findbücher bis heute orientieren, war Karl Kaufmann ein Sinto aus Süddeutschland, und Pauline Reinhardt wurde als "Zigeunerartige“ kategorisiert. Weitere Hinweise auf das Leben der beiden mehrfach Abgelichteten sind in den Karteien nicht vorhanden und konnten nicht ermittelt werden. ${ }^{32}$

Diese Art der fotografischen Darstellung gibt es bis heute. Sie hat sich international durchgesetzt und ruft bei den Betrachter_innen die Assoziation „kriminell“ hervor. Unter den Begriffen „Verbrecherbild“, „Verbrechergesicht" und „mug shot" gibt es Studien dazu, dass Personen, die in diesem Format abgelichtet werden, für Straftäter_innen gehalten werden, und zwar unabhängig davon, ob sie tatsächlich Straftaten begangen haben. ${ }^{33}$ Die Trias des Verbrecherfotos wirkt insofern als Drohszenario, als allein die Darstellung in diesem Format abschreckend wirken soll und tatsächlich wirkt. Die Abbildung in dieser Form suggeriert, dass die Person ein Verbrechen begangen hat. Dies trifft Angehörige einer Minderheit, die bis heute unter einem pauschalen Verdacht steht und daher überdurchschnittlich häufig von polizeilichen Institutionen erfasst wird, in besonderem Maße und wirkt - einmal angestoßen - selbstverstärkend. Denn je mehr Personen einer Gruppe erfasst werden, desto stärker steht sie im Fokus der Ermittlungsbehörden, und desto mehr Gruppenangehörige werden wiederum erfasst.

und bei denen es sich um Privatfotografien handeln könnte. Anders als auf den standardisierten Fotografien, ist Pauline Reinhardt auf einem der Bilder mit ihrer 1929 geborenen Enkelin zu sehen, deren Hand sie hält. Ihr Blick ist der Kamera zugewandt und zeigt ein Lächeln. Sie ist vor einem, eventuell ihrem eigenen Wohnwagen zu sehen. Die Bilder wurden wahrscheinlich an verschiedenen Tagen aufgenommen, da Pauline Reinhardt unterschiedliche Kleidung trägt. Aus Gründen des Personenschutzes der Enkelin werden die Fotografien nicht abgedruckt.

32 Eine Frau Namens Pauline Reinhardt aus Pfullendorf-Otterswang, allerdings ein Jahr später geboren als die hier verzeichnete, war unter denjenigen, die am 15.3.1943 vom Stuttgarter Nordbahnhof aus in das sogenannte "Zigeunerlager" in Auschwitz-Birkenau deportiert wurden, vgl. Verband Deutscher Sinti und Roma, Landesverband Baden-Württemberg (2018, S. 36). Die meisten der Deportierten starben dort.

33 Jens Jäger spricht von der „Gleichung Identifikationsfoto $=$ Krimineller“ (2009, S. 376). 


\section{Schlussfolgerungen}

Auf die Fragen, wie sich das Bild des kriminellen „Zigeuners“ oder der kriminellen „Zigeunerin“ im doppelten Sinne als Vorstellung und als tatsächliches bildliches Motiv etabliert hat und durch staatliche Institutionen, insbesondere die Polizei, eingesetzt wurde, lässt sich in Anbetracht der analysierten Bilder und Fotografien Folgendes zusammenfassen: Die ausgewählten Fallbeispiele zeigen, dass der gezielte Einsatz von Bildern durch staatliche Institutionen zur Versicherheitlichung der Sinti_ze und Rom_nja eine jahrhundertelange Tradition hat und nicht erst mit der Fotografie begann. Bereits im 17. Jahrhundert wurden Menschen, die unter dem Begriff „Zigeuner" stigmatisiert wurden, für eine breite Bevölkerungsschicht, die nicht des Lesens mächtig sein musste, visuell als unerwünschte und zu bestrafende Gruppe dargestellt. Dies geschah am Wegesrand, an Grenzübergängen und vor Stadttoren über die weit verbreiteten „Zigeunerwarntafeln“, die einen präventiven Charakter in Bezug auf Gesetzesbrüche haben sollten. Sie adressierten sowohl die direkt angesprochene Gruppe der Umherziehenden als auch die übrige Bevölkerung und repräsentierten ein Drohszenario für einen abweichenden Lebensstil. Ferner wurden tatsächlich durchgeführte Prozesse und Hinrichtungen spektakulär auch bildlich inszeniert, wie das Buch über den Gießener Gerichtsprozess zu Beginn des 18. Jahrhunderts offenbart.

Weiterhin konnte gezeigt werden, dass sich die visuelle Versicherheitlichung im Verlaufe der Jahrhunderte auf mehreren Ebenen wandelte. Erstens veränderte sich die Art der visuellen Markierung der zu Bestrafenden. Während die im Bild angedrohten und in der Realität vollzogenen körperlichen Strafen wie Brandmarkung oder abgetrennte Gliedmaßen die Bestraften sichtbar kennzeichneten, wurde mit der Fotografie zunehmend der Aspekt der Wiedererkennbarkeit der Personen im Bild relevant.

Damit einhergehend vollzog sich zweitens ein Wandel in Bezug auf die Strafandrohung. Während in den vor-fotografischen Bildern die drohende Strafe explizit gemacht wurde, ist eine Strafandrohung bei den Polizeifotografien nicht auf den ersten Blick erkennbar. Erst indem die gesellschaftliche Wirkung und Einbettung der Fotografien einbezogen und die Prozesse einer Standardisierung der fotografischen Ästhetik analysiert werden, wird deutlich, dass durch die fotografische Aufnahme der Individuen in einem bestimmten und fixierten Rahmen eine ganze Bevölkerungsgruppe als verdächtig und kriminell visualisiert wird. Das Drohszenario der frühen gemalten Bilder bleibt demnach erhalten, transformiert sich aber in eine subtilere Form. 
Damit veränderte sich drittens auch die Art der Darstellung der Devianz. In den frühen Bildern sind die zu bestrafenden Personen hauptsächlich im Gegensatz zu den Vertretern des Staates und der Kirche dargestellt. In der Dreier-Reihe des Verbrecherbildes wirken hingegen auf den ersten Blick alle gleich. Wiederum unter Einbezug der Entstehungsgeschichte lässt sich jedoch zeigen, dass die Standardisierung des Verbrecherbildes gerade in Abgrenzung zur bürgerlichen Porträt-Fotografie geschah. Parallel zu dieser allgemeinen Entwicklung der erkennungsdienstlichen Fotografie, die aus Kostengründen zu Beginn des 20. Jahrhunderts hauptsächlich für Serienstraftäter_innen benutzt wurde, wurden zu dieser Zeit tausende Fotografien von Sinti_ze, Rom_nja und anderen als „Zigeuner“ oder „Zigeunerin" Klassifizierten nach demselben Muster aufgenommen. Unabhängig davon, ob sie tatsächlich Straftaten begangen hatten, wurden Frauen, Männer und Kinder in die polizeilichen Karteien eingespeist und so behandelt, als wäre bereits ihre Existenz eine Straftat. Für die Betroffenen wurde somit bereits die permanente Bedrohung der fotografischen Erfassung zu einer subtilen Strafandrohung, bedeutete doch die Aufnahme und Veröffentlichung des Bildes eine gesellschaftliche Stigmatisierung und Ausgrenzung. Angelehnt an Foucaults Analyse des Wandels von körperlichen Bestrafungen hin zum Freiheitsentzug als Strafe der modernen Gesellschaft (vgl. Foucault 1989, S. 291), kann die Standardisierung der Fotografien nicht nur als Versicherheitlichung, sondern auch als Akt der Disziplinierung durch visuelle Methoden bezeichnet werden. ${ }^{34}$

Nicht zuletzt lässt sich anhand der technischen und inhaltlichen Entwicklung der frühen Polizeifotografien erkennen, dass in den Bildern die außerbildliche Wirklichkeit zunehmend reduziert wird und eine Konzentration auf das Gesicht und die Physiognomie stattfindet. Dies geschah unter anderem durch die Verkleinerung des fotografischen Ausschnitts auf Bruststück-Ansichten. Die Fotografien entsprechen damit den von Susan Sontag beschriebenen Bedürfnissen der modernen, kapitalistischen Gesellschaft nach unkomplizierten und widerspruchsfreien Bildern. Sie kreieren qua Vergleichbarkeit zugleich Unterschiede und Gleichheit - vermeintli-

34 Auch Foucault verwendete für seine Analysen teilweise Bildmaterial, mit Polizeifotografie beschäftigte er sich jedoch nicht. Die Frage, ob Foucault Bilder systematisch in seine Diskursanalyse einbezog, ist Gegenstand der aktuellen Forschungsdebatte im Bereich der historischen Diskursanalyse. Vgl hierzu etwa Zittel (2014, S. 87), der keinen systematischen Einbezug bei Foucault erkennen kann, und Renggli (2014, S. 49), die hingegen stark macht, dass genau die wechselseitigen Beziehungen von Sprache, Dingen und Bildern das Zentrum von Foucaults Diskursanalyse bilden. 
che Differenz in Hinblick auf die abgebildete gesellschaftliche Gruppe, scheinbare Homogenität unter den Abgebildeten. Gesellschaftliche Spannungen und Widersprüche werden somit ausgeblendet, während zugleich Hierarchien (re-)produziert werden. Dies geschieht indem sich staatliche Institutionen als stark und mächtig inszenieren - zunächst im gemalten Bild als strafende Repräsentanten des Staates, später außerhalb des Bildes als rahmensetzende Instanz, die zunehmend die alleinige Entscheidungshoheit über die Darstellung hat. Die stark normierten Fotografien sollen Effektivität und Kontrolle demonstrieren und dienen letztlich auch als (Selbst-)Rechtfertigung des polizeilichen Vorgehens.

\section{Literatur}

\section{Archive}

BArch - Deutschen Bundesarchiv Berlin

CH-BAR - Schweizerisches Bundesarchiv Bern

StadAM - Stadtarchiv München

StAM - Staatsarchiv München

\section{Quellen}

Dillmann, Alfred (1905): Zigeuner-Buch. Herausgegeben zum amtlichen Gebrauche im Auftrage des K. B. Staatsministeriums des Innern vom Sicherheitsbureau der K. Polizeidirektion München. München: Dr. Wild'sche Buchdruckerei.

Grellmann, Heinrich Moritz Gottlieb (1783): Die Zigeuner. Ein historischer Versuch über die Lebensart und Verfassung, Sitten und Schicksahle dieses Volkes in Europa, nebst ihrem Ursprunge. Dessau, Leipzig.

Weissenbruch, Johann Benjamin (1727): Ausführliche Relation Von der Famosen Ziegeuner-, Diebs-, Mord- und Rauber-Bande, Welche Den 14. und 15. Novembr. Ao. 1726. zu Giessen durch Schwerdt, Strang und Rad, respective justificirt worden. Worinnen Nach praemittirter Historie von dem Ursprung und Sitten derer Zigeuner [et]c. [et]c. die vornehmste und schwereste Begangenschafften mit allen Umständen erzehlet, auch was durante Processu sowol ante- als in$\&$ post Torturam vorgenommen worden, enthalten ist, Aus denen weitläufftigen Peinlichen Original-Actis in möglichster Kürtze zusammen gezogen, Und auf Sr. Hochfürstl. Durchl. zu Hessen-Darmstadt Gnädigste Special-Erlaubnüß, Dem Publico zum Besten, in öffentlichen Druck befördert; Mit einigen Kupffern. Leipzig, [Marburg], [Giessen], Franckfurt: Krieger. 
Welcker, Adolph (4./5.2.1902): Die Zigeunerplage. In: Darmstädter Zeitung: amtliches Organ der Hessischen Landesregierung 126, Beilagen zu Nr. 58 und 60, S. 235 und 243.

\section{Sekundärliteratur}

Agarin, Timofey (Hrsg.) (2014): When Stereotype Meets Prejudice: Antiziganism in European Societies. Stuttgart: ibidem.

Albrecht, Angelika (2002): Zigeuner in Altbayern: 1871-1914. Eine sozial-, wirtschafts- und verwaltungsgeschichtliche Untersuchung der bayerischen Zigeunerpolitik. München: Kommission für bayerische Landesgeschichte. (Zugl.: Regensburg, Univ., Diss., 1998).

Amaro Foro e.V. (Hrsg.) (2018): Dokumentation antiziganistischer und diskriminierender Vorfälle in Berlin 2017. Berlin: Eigenverlag.

Bell, Peter (2015): Fataler Blickkontakt. Wie in „Zigeunerbildern“ Vorurteile inszeniert werden. In: Dokumentations- und Kulturzentrum Deutscher Sinti und Roma/Baumann, Thomas (Hrsg.): Antiziganismus. Soziale und historische Dimensionen von „Zigeuner"-Stereotypen. Heidelberg: Dokumentations- und Kulturzentrum Dt. Sinti und Roma, S. 150-167.

Benz, Wolfgang (2014): Sinti und Roma: Die unerwünschte Minderheit. Über das Vorurteil Antiziganismus. Berlin: Metropol.

End, Markus (2019). Antiziganismus und Polizei. Mit Dokumentation der Fachveranstaltung "Die Polizei und Minderheiten - Das Beispiel Antiziganismus" und einem ergänzenden Beitrag zum OEZ-Attentat. Heidelberg: Eigenverlag. (Herausgegeben vom Zentralrat Deutscher Sinti und Roma).

Fings, Karola (2008): »Rasse: Zigeuner«. Sinti und Roma im Fadenkreuz von Kriminologie und Rassenhygiene 1933-1945. In: Uerlings, Herbert/Patrut, Iulia-Karin (Hrsg.): ,Zigeuner ${ }^{6}$ und Nation. Repräsentation - Inklusion - Exklusion. Frankfurt am Main: Peter Lang, S. 273-309.

Foucault, Michel (1989): Überwachen und Strafen. Die Geburt des Gefängnisses. 8. Aufl, Frankfurt am Main: Suhrkamp.

Fricke, Thomas (1996): Zigeuner im Zeitalter des Absolutismus. Bilanz einer einseitigen Überlieferung. Eine sozialgeschichtliche Untersuchung anhand süddeutscher Quellen. Pfaffenweiler: Centaurus (zugl.: Tübingen, Univ., Diss., 1994/95).

Gasser, Martin (1998): Bodenständig und heimatlos. Carl Durheim, Erich Stenger und die "photographierten Schweizer Vaganten". In: Gasser, Martin/Meier, Thomas Dominik/Wolfensberger, Rolf (Hrsg.): Wider das Leugnen und Verstellen. Carl Durheims Fahndungsfotografien von Heimatlosen 1852/53. Winterthur, Zürich: Fotomuseum Winterthur, S. 125-141.

Geimer, Peter (2010): Theorien der Fotografie zur Einführung. 2., verb. Aufl., Hamburg: Junius. 
Giere, Jacqueline (Hrsg.) (1996): Die gesellschaftliche Konstruktion des Zigeuners. Zur Genese eines Vorurteils. Frankfurt am Main, New York: Campus.

Härter, Karl (2005): Recht und Migration in der frühneuzeitlichen Ständegesellschaft. Reglementierung - Diskriminierung - Verrechtlichung. In: Beier-de Haan, Rosmarie (Hrsg.): Zuwanderungsland Deutschland. Migrationen 15002005. Wolfratshausen, Berlin: Minerva; DHM, S. 50-71.

Hessisches Kultusministerium (Hrsg.) (2015): Sinti und Roma in Deutschland und die Rolle des Antiziganismus. Handreichung für den fächerübergreifenden Unterricht. Wiesbaden.

Heuß, Herbert (2003): Aufklärung oder Mangel an Aufklärung? Über den Umgang mit den Bildern vom „Zigeuner“. In: Engbring-Romang, Udo (Hrsg.): Aufklärung und Antiziganismus. Beiträge zur Antiziganismusforschung. Seeheim: IVerb.de, 11-33.

Holzer, Anton (2008): Faszination und Abscheu. Die fotografische Erfindung der Zigeuner. In: Fotogeschichte. Beiträge zur Geschichte und Ästhetik der Fotografie 28 , H. 110, S. 45-56.

Huonker, Thomas/Ludi, Regula (2001): Roma, Sinti und Jenische. Schweizerische Zigeunerpolitik zur Zeit des Nationalsozialismus. Zürich: Chronos.

Illuzzi, Jennifer (2014): Gypsies in Germany and Italy, 1861-1914. Lives Outside the Law. Basingstoke, Hampshire: Palgrave Macmillan.

Jäger, Jens (2006): Verfolgung durch Verwaltung. Internationales Verbrechen und internationale Polizeikooperation 1880-1933. Konstanz: UVK. (Zugl.: Köln, Univ., Habil.-Schr., 2004).

Jäger, Jens (2009): „Verbrechergesichter“. Zur Geschichte der Polizeifotografie. In: Paul, Gerhard (Hrsg.): Das Jahrhundert der Bilder. 1900 bis 1949. Göttingen: Vandenhoeck \& Ruprecht, S. 372-379.

Jeutter, Ewald (2016): Das Verbrecherbildnis von der Frühen Neuzeit bis 1850. Sammelstück und mediale Ausprägung. In: Krems, Eva-Bettina/Ruby, Sigrid (Hrsg.). Das Porträt als kulturelle Praxis. Berlin, München: Deutscher Kunstverlag, S. 32-44.

Kammerer, Dietmar (2007): Welches Gesicht hat das Verbrechen? Die "bestimmte Individualität" von Alphonse Bertillons "Verbrecherfotografie". In: Zurawski, Nils (Hrsg.): Sicherheitsdiskurse. Angst, Kontrolle und Sicherheit in einer "gefährlichen“ Welt. Frankfurt am Main: Peter Lang, S. 27-37.

Koch, Ute (2010): Soziale Konstruktion und Diskriminierung von Sinti und Roma. In: Hormel, Ulrike/Scherr, Albert (Hrsg.): Diskriminierung. Grundlagen und Forschungsergebnisse. Wiesbaden: VS Verlag für Sozialwissenschaften, S. 255278.

Landwehr, Achim (2001): Norm, Normalität, Anomale. Zur Konstitution von Mehrheit und Minderheit in württembergischen Policeyordnungen der Frühen Neuzeit: Juden, Zigeuner, Bettler, Vaganten. In: Häberlein, Mark/Zürn, Martin (Hrsg.): Minderheiten, Obrigkeit und Gesellschaft in der frühen Neuzeit. Integrations- und Abgrenzungsprozesse im süddeutschen Raum. St. Katharinen: Scripta Mercaturae Verlag, S. 41-74. 
Leimgruber, Walter (2005): Die visuelle Darstellung des menschlichen Körpers. Gesellschaftliche Aus- und Eingrenzungen in der Fotografie. In: Gerndt, Helge/ Haibl, Michaela (Hrsg.): Der Bilderalltag. Perspektiven einer volkskundlichen Bildwissenschaft. Münster: Waxmann, S. 213-232.

Lucassen, Leo (1996): Zigeuner. Die Geschichte eines polizeilichen Ordnungsbegriffes in Deutschland 1700-1945. Köln, Weimar, Wien: Böhlau.

Luchterhandt, Martin (2007): Robert Ritter und sein Institut. Vom Nutzen und Benutzen der "Forschung". In: Zimmermann, Michael (Hrsg.): Zwischen Erziehung und Vernichtung. Zigeunerpolitik und Zigeunerforschung im Europa des 20. Jahrhunderts. Stuttgart: Steiner, S. 321-328.

Mappes-Niediek, Norbert (2013): Arme Roma, böse Zigeuner. Was an den Vorurteilen über die Zuwanderer stimmt. 3., durchges. Aufl., Berlin: Ch. Links Verlag.

Meier, Thomas (2007): Zigeunerpolitik und Zigeunerdiskurs in der Schweiz 1850 1970. In: Zimmermann, Michael (Hrsg.): Zwischen Erziehung und Vernichtung. Zigeunerpolitik und Zigeunerforschung im Europa des 20. Jahrhunderts. Stuttgart: Steiner, S. 226-239.

Meier, Thomas Dominik/ Wolfensberger, Rolf (1998a): Carl Durheims Fahndungsfotografien von schweizerischen Heimatlosen und Nicht-Sesshaften. In: Gasser, Martin/Meier, Thomas Dominik/ Wolfensberger, Rolf (Hrsg.): Wider das Leugnen und Verstellen. Carl Durheims Fahndungsfotografien von Heimatlosen 1852/53. Winterthur, Zürich: Fotomuseum Winterthur, S. 9-24.

Meier, Thomas Dominik/Wolfensberger, Rolf (1998b): «Eine Heimat und doch keine». Heimatlose und Nicht-Sesshafte in der Schweiz (16.-19. Jahrhundert). Zürich: Chronos.

Meuser, Maria (2014): Vagabunden und Arbeitsscheue. Der Zigeunerbegriff der Polizei als soziale Kategorie. In: Hund, Wulf D. (Hrsg.): Faul, fremd und frei. Dimensionen des Zigeunerstereotyps. Neuaufl. in einem Bd., Münster: Unrast, S. 105-123.

Opfermann, Ulrich Friedrich (2016): Zur Lage der Roma in Deutschland von der Reichsgründung 1871 bis 1933. In: Nerdinger, Winfried (Hrsg.): Die Verfolgung der Sinti und Roma in München und Bayern 1933-1945. Publikation zur Ausstellung im NS-Dokumentationszentrum München 27. Oktober 2016 bis 29. Januar 2017. Berlin: Metropol, S. 56-69.

Regener, Susanne (1999): Fotografische Erfassung. Zur Geschichte medialer Konstruktionen des Kriminellen. München: Wilhelm Fink.

Renggli, Cornelia (2014): Komplexe Beziehungen beschreiben. Diskursanalytisches Arbeiten mit Bildern. In: Eder, Franz X./Kühschelm, Oliver/Linsboth, Christina (Hrsg.): Bilder in historischen Diskursen. Wiesbaden: Springer VS, S. 45-61.

Reuter, Frank (2011): Fotografische Repräsentation von Sinti und Roma. Voraussetzungen und Traditionslinien. In: Peritore, Silvio/Reuter, Frank (Hrsg.): Inszenierung des Fremden. Fotografische Darstellung von Sinti und Roma im Kontext der historischen Bildforschung. Heidelberg: Dokumentations- und Kulturzentrum Dt. Sinti und Roma, S. 163-221. 
Reuter, Frank (2014): Der Bann des Fremden. Die fotografische Konstruktion des Zigeuners. Göttingen: Wallstein.

Reuter, Frank (2016): Der selektive Blick. Die fotografische Konstruktion des "Zigeuners". In: Nerdinger, Winfried (Hrsg.): Die Verfolgung der Sinti und Roma in München und Bayern 1933-1945. Publikation zur Ausstellung im NS-Dokumentationszentrum München 27. Oktober 2016 bis 29. Januar 2017. Berlin: Metropol, S. 28-39.

Reuter, Frank (2019): Gesichtslos. Kontinuitäten antiziganistischer Wahrnehmungsmuster. In: Brunner, Andreas/ Staudinger, Barbara/ Sulzenbacher, Hannes/ Zadoff, Mirjam (Hrsg.): Die Stadt ohne. Juden Ausländer Muslime Flüchtlinge. München: Hirmer Verlag, S. 185-189.

Schmitz, Manfred-Guido (Hrsg.) (2011): Der Justiz-Skandal am Peinlichen Gericht zu Giessen. "Ausführliche Relation von der famosen Ziegeuner-, Diebs-, Mordund Rauber-Bande" (1727) von Dr. jur. Johann Benjamin Weissenbruch in einer kommentierten Überarbeitung. Nordstrand: M.-G. Schmitz-Verlag.

Solms, Wilhelm (2008): Zigeunerbilder. Ein dunkles Kapitel der deutschen Literaturgeschichte. Von der frühen Neuzeit bis zur Romantik. Würzburg: Königshausen \& Neumann.

Sontag, Susan (1987): Über Fotografie. Frankfurt am Main: Fischer Taschenbuch Verlag.

Steiner, Stephan (2019): The Enemy Within. 'Gypsies' as EX/INternal Threat in the Habsburg Monarchy and in the Holy Roman Empire, $15^{\text {th }}-18$ th Century. In: Crailsheim, Eberhard/Pérez-Grueso, Elizalde/Dolores, María (Hrsg.): The Representation of External Threats. From the Middle Ages to the Modern World. Leiden, Boston: BRILL, S. 131-154.

Tittel, Laura Soréna (2020): Racial and Social Dimensions of Antiziganism. The Representation of "Gypsies" in the Political Theory. In: On_Culture: The Open Journal for the Study of Culture, H. 10, S. 1-23. geb.uni-giessen.de/geb/volltexte/2021/16025/, 15.05.2021.

Uerlings, Herbert/Trauth, Nina/Clemens, Lukas (Hrsg.) (2011): Armut. Perspektiven in Kunst und Gesellschaft. Darmstadt: Primus Verlag.

Vec, Miloš (2002): Die Spur des Täters. Methoden der Identifikation in der Kriminalistik (1879-1933). Baden-Baden: Nomos.

Verband Deutscher Sinti und Roma, Landesverband Baden-Württemberg (2018): „Ich kann das nicht begreifen.“ Dokumentation zu 75 Jahre Gedenken an die Deportationen von Sinti und Roma aus Baden-Württemberg. www.gedenkstaett en-bw.de/fileadmin/gedenkstaetten/pdf/publikationen/doku_75jahre_gedenk_si nti_roma.pdf, 10.05.2021.

Wieckhorst, Egon (2012): Zur Geschichte des Wülfinger Taternpfahles von 1635. In: Förderverein für die Stadtgeschichte von Springe e.V. (Hrsg.): Springer Jahrbuch 2012 für die Stadt und den Altkreis Springe. Springe: Eigenverlag, S. 100106.

Willems, Wim (1997): In Search of the True Gypsy. From Enlightenment to Final Solution. London, Portland, OR: Frank Cass. 
Wippermann, Wolfgang (2015): Niemand ist ein Zigeuner. Zur Ächtung eines europäischen Vorurteils. Hamburg: Edition Körber-Stiftung.

Zittel, Claus (2014): Die Ordnung der Diskurse und das Chaos der Bilder. Bilder als blinde Flecken in Foucaults Diskursanalyse und in der Historiographie der Philosophie? In: Eder, Franz X./Kühschelm, Oliver/Linsboth, Christina (Hrsg.): Bilder in historischen Diskursen. Wiesbaden: Springer VS, S. 85-107. 
